Archives

\title{
Élucider et équivoquer : Francesco Robortello (ré)invente la « catharsis »
}

\section{Déborah Blocker}

\section{(2) OpenEdition}

Journals

\section{Édition électronique}

URL : http://journals.openedition.org/ccrh/250

DOI : $10.4000 /$ ccrh.250

ISSN : $1760-7906$

Éditeur

Centre de recherches historiques - EHESS

Édition imprimée

Date de publication : 1 avril 2004

ISSN : 0990-9141

Référence électronique

Déborah Blocker, «Élucider et équivoquer : Francesco Robortello (ré)invente la « catharsis » », Les Cahiers du Centre de Recherches Historiques [En ligne], 33 | 2004, mis en ligne le 19 novembre 2008, consulté le 10 décembre 2020. URL : http://journals.openedition.org/ccrh/250 ; DOI : https://doi.org/ $10.4000 /$ ccrh. 250

Ce document a été généré automatiquement le 10 décembre 2020.

Article L.111-1 du Code de la propriété intellectuelle. 


\title{
Élucider et équivoquer : Francesco Robortello (ré)invente la « catharsis »
}

\author{
Déborah Blocker
}

\section{NOTE DE L'AUTEUR}

Je tiens à remercier Marwan Rashed pour son aide dans l'élucidation du texte de Robortello, ainsi que l'ensemble du Warburg Institute, où une grande partie des recherches nécessaires à la rédaction de cet article ont été effectuées, lors de mon séjour en tant que Brian Hewson Crawford Fellow au printemps 2003. Au sein de cette dernière institution, les remarques de Charles Burnett, Helen Dixon, Jill Kraye, François Quiviger et Anna Somfai me furent particulièrement précieuses. Dinah Ribard fut, comme toujours, ma relectrice la plus intransigeante. Elle ne sera jamais assez remerciée. Les critiques de Pierre Judet de la Combe m'ont permis de mieux cerner les textes d'Athénée, de Plutarque et de Proclus. Mes collègues de l'université de Pittsburgh, Dennis Looney et Daniel Russell, m'ont prodigué d'ultimes suggestions. Enfin, Sandrine Lély m'a relue avec la plus extrême attention.

En mémoire de Stéphane Diebler

1 «[...] Quce alii rejecerant propter obscuritatem, ea ego illustrare me posse consider[o]» («ces textes que d'autres ont repoussé en raison de leur obscurité, je me tiens pour capable de les élucider »), clame Francesco Robortello (1516-1567) aux premières lignes de ses In Librum De Arte Poetica Explicationes, parues à Florence en 1548 et dédiées à Cosme I ${ }^{\mathrm{er}}$ de Médicis'. En se vantant de pouvoir éclaircir un texte dont la complexité aurait découragé tous ses prédécesseurs, l'auteur du premier commentaire de la Poétique d'Aristote à avoir trouvé le chemin de l'impression adoptait une posture très répandue chez les humanistes de l'Italie des $\mathrm{Xv}^{\mathrm{e}}$ et $\mathrm{XvI}^{\mathrm{e}}$ siècles ${ }^{2}$. La concurrence pour l'édition ou le commentaire des textes anciens y était féroce, puisque devancer ses rivaux dans la publication ou l'élucidation d'une œuvre antique était, pour qui désirait s'attirer l'attention des patrons 
les plus prestigieux ou obtenir les postes universitaires les plus en vue, un titre de gloire qui pouvait ouvrir le chemin des meilleures places ${ }^{3}$. La proclamation tonitruante de Robortello est d'autant plus révélatrice qu'elle lie son désir de briller - en éclipsant tous les concurrents éventuels - aux principes fondateurs de la philologie humaniste ellemême, soit à la prétention de mettre au jour et en lumière les textes anciens, en redonnant sens et éclat à leurs phrases tenues pour corrompues par le temps et les scribes. La philologie héritée de Lorenzo Valla et d'Ange Politien se définissait principalement par cette ambition de clarifier, d'éclaircir et d'élucider. Par la publication, la traduction et le commentaire, la pensée des anciens devait entrer dans la lumière. Dans le cas du commentaire de la Poétique par Robortello, cette ambition, doublée d'une visée pédagogique, s'afficha jusque dans le sous-titre de l'ouvrage qui présentait son auteur comme un élucidateur capable de mettre les ouvrages les plus abscons à la portée de tout un chacun. Le texte de la Poétique était ainsi décrit comme "amendé par l'auteur en de nombreux lieux d'après les manuscrits [eux-mêmes], afin que ce livre [tenu] jusqu'ici [pour] des plus difficiles et des plus obscurs-et jamais encore commenté par quiconque -, puisse être facilement compris par tout le monde». Et si l'ambition d'élucider était généralement indissociable du désir de briller et, par là, de parvenir, c'était d'abord parce qu'en illustrant les textes des Anciens de gloses qui se prétendaient lumineuses, le philologue illustrait avant tout sa propre habileté - tout en donnant à voir, le cas échéant, la clairvoyance de son patron. Selon le double sens du latin illustrare, le lustre donné au texte devait rejaillir sur l'interprète, et sur l'éventuel commanditaire.

\section{La philologie entre érudition et carriérisme}

2 Cependant, quoique les ambitions sociales et institutionnelles des humanistes se soient le plus souvent remarquablement bien accordées avec l'ethos philologique qui faisait de l'élucidation des textes anciens une valeur suprême, il arrivait aussi que cet idéal de clarté, constamment réaffirmé dans les écrits liminaires des textes élucidés, rentre en conflit avec les conditions politiques dans lesquelles les humanistes exerçaient alors leur (s) profession(s). Une carrière en tant que professeur d'humanités grecques et latines dans les lieux de savoir de l'Italie du Cinquecento exigeait des lettrés qui s'y risquaient autant de connaissances philologiques que de savoir-faire politique. Tout d'abord parce que, le plus souvent, ces "carrières" se faisaient et se défaisaient en dehors des universités et académies qui les accueillaient en leur sein. Les pouvoirs locaux, dont ces lieux de savoir dépendaient, exerçaient un droit de sélection et/ou de nomination pour la plupart des chaires à pourvoir, soit directement, soit indirectement. Ceci était tout particulièrement vrai dans les humanités, où l'enseignant n'avait pas pour seule charge celle d'enseigner à ses élèves l'interprétation des textes anciens. Dans l'esprit des autorités politiques qui les appointaient, aussi bien que dans certaines des représentations que les humanistes véhiculaient de leurs propres activités, la pratique des humanités avait pour fonction sociale essentielle de former moralement et politiquement les élites (présentes tout autant que futures) d'une cité à l'exercice des charges dirigeantes.

3 En raison de ces finalités sociales et politiques, les carrières des humanistes furent souvent beaucoup plus polyvalentes-et, de ce fait même, parfois beaucoup plus évolutives -, que celle des enseignants chargés de professer la philosophie naturelle ou la théologie. Commençant leur cursus honorum en tant que lecteurs de logique et/ou 
d'humanités grecques et latines ${ }^{4}$, les humanistes terminèrent de plus en plus souvent leurs carrières, au XVI ${ }^{\mathrm{e}}$ siècle, comme professeurs de philosophie morale et politique ${ }^{5}$. Ce parcours fut notamment celui de Francesco Robortello. Chargé d'enseigner la logique et les humanités à Pise dès 1543, il y commenta aussi les œuvres rhétoriques et morales de Cicéron à partir de 1547. En 1549, il s'installa à Venise, où il exposa d'abord l'éthique aristotélicienne, avant de s'attaquer, dès 1550, à un commentaire de la Politique. À vrai dire, un tel parcours intellectuel, qui le conduisit, à la fin de sa carrière, à la chaire de philosophie morale et politique de l'université de Padoue, était, en 1540-1550, relativement inhabituel dans sa manière d'articuler philologie, poétique, rhétorique, morale et politique : n'étaient quelques pionniers tels qu'Ange Politien ou Giorgio Valla, on pourrait même dire que Robortello fut un précurseur. Il semble néanmoins plus important de souligner que l'homme fit immédiatement des émules: nombre des meilleurs humanistes de l'Italie du Nord lui emboîtèrent très rapidement le pas ${ }^{6}$.

4 À cette pluridisciplinarité apparente - apparente dans le sens où cette activité ne paraît pluridisciplinaire à nos yeux de modernes que parce nous évoluons, depuis le XIX siècle, au sein d'une conception étroitement disciplinarisée des studia humanitatis - s'ajoute souvent une pluriactivité. Celle-ci se marque notamment par le fait que, au sein même de la cité, les humanistes délivrent leurs enseignements ou exhortations en des lieux très divers. En plus de son enseignement régulier à l'université, située parfois en dehors des murs de la cité - le principal centre universitaire florentin se trouvait à Pise, tandis que l'université de Padoue était le plus important lieu d'enseignement et de recherche de la Vénétie - un professeur d'humanités pouvait aussi se voir confier l'oraison funèbre d'un haut personnage (à prononcer dans le duomo), des conférences d'apparat à donner en italien au sein d'une des académies locales ou encore une charge de cours au sein des scuole spécialisées, mises en place dans la cité même, pour former les secrétaires et autres personnels de chancellerie dont l'administration de la ville pouvait avoir besoin.

Robortello ne négligea aucune de ces activités para-universitaires, dans l'exercice desquelles il espérait pouvoir séduire les puissants ${ }^{7}$. À la fin de ses études à l'université de Bologne, il obtint rapidement - à la fin de l'année 1537 ou peut-être au début de l'année 1538 - un poste de lecteur en humanités et en rhétorique au sein de la petite université de Lucques, dans la périphérie politique de Florence. Une fois dans cette ville, il s'empressa $\mathrm{d}$ 'accepter les oraisons funèbres qui lui furent confiées par l'aristocratie locale ${ }^{8}$. Sous le dôme de la cathédrale et devant l'ensemble de la bonne société de la ville, il trouva ainsi la possibilité de démontrer tout à la fois l'ampleur de ses talents de rhéteur et l'orthodoxie de ses positions en matière de philosophie morale et politique. Nul doute que ces interventions lui permirent de se faire connaître des érudits comme des notables les plus influents à Florence, le positionnant pour décrocher un poste à Pise dès que le Studio Pisano rouvrit ses portes, en 1543. Quelques lettres éparses datant de ces années passées à Lucques nous montrent Robortello étroitement lié à Francesco Verino et à Pier Vettori ${ }^{9}$. Or le vieux philosophe Francesco Verino s'était rallié très vite au pouvoir de Cosme de Médicis, que l'assassinat de son lointain cousin Alessandro avait porté au pouvoir de manière inattendue en $1537^{10}$. C'était à lui qu'on avait fait appel pour prononcer, en février 1541, la première leçon publique de l'Académie Florentine, à laquelle deux mille notables florentins auraient assisté. Pier Vettori n'était pas moins proche du nouveau pouvoir. Appartenant à une ancienne famille patricienne, il avait fui la ville lorsque Cosme avait été élu à sa tête, mais rentra à Florence après la bataille de Montemurlo, qui vit la défaite des opposants au clan des Médicis. Une correspondance témoigne des liens 
étroits de Vettori avec Francesco Campana ${ }^{11}$, l'influent premier secrétaire de Cosme, qui fut notamment chargé de sélectionner les lettrés susceptibles de professer dans les lieux de savoirs toscans, que les hommes du duc s'attachaient à faire prospérer de nouveau. Or Campana est aussi l'homme qui fit élire Robortello au Studio Pisano, sur un poste très similaire à celui qu'il avait offert à Vettori à Florence, dans le cadre du Studio Florentino, - somptueuse rétribution mise à part ${ }^{12}$.

Cette élection constitua probablement une promotion inespérée pour Robortello. Certes, celui-ci venait de publier à Venise son premier ouvrage, présentant la résolution d'une série de difficultés philologiques, tirées de ses cours d'humanités latines et grecques à Lucques. Le livre eut même quelque succès, puisqu'une édition parut à Paris dès l'année suivante et qu'une seconde édition, augmentée d'un second livre contenant les remarques nées de ses cours à Pise, fut publiée à Florence en 1548, chez l'éditeur privilégié du grand duc, Lorenzo Torrentino ${ }^{13}$. Mais ce genre de travaux, quoique prisés des humanistes et susceptibles d'attirer à un philologue l'estime de ses pairs, pouvait difficilement suffire à faire naître la bienveillance d'un puissant personnage. Seules les oraisons funèbres et autres interventions publiques devant les représentants des classes dirigeantes permettaient d'obtenir de telles protections. Et c'est sans doute en raison de ces activités parallèles que, lorsque Giambattista Adriani, à qui le poste de Pise avait d'abord été destiné, y renonça, les amis de Robortello purent le recommander aux conseillers de Cosme qui l'élirent en septembre 1543, sur proposition de Francesco Campana ${ }^{14}$. Une fois nommé à Pise, Robortello continua à prêter une attention extrême aux activités parauniversitaires, qui influèrent parfois considérablement sur le choix de ses objets d'étude.

7 Ainsi s'explique, du moins pour une part, son choix de publier le premier commentaire extensif de la Poétique d'Aristote. Car, alors que le platonisme avait été une tradition à Pise comme à Florence depuis Marsile Ficin - Laurent le Magnifique l'ayant toujours favorisé - , au moment où Robortello s'attela à son commentaire, le pouvoir médicéen encourageait les lettrés qui le servaient à s'appuyer plutôt sur Aristote. Aux yeux de Cosme et de son entourage, Platon, que leurs opposants revendiquaient pour justifier leurs convictions républicaines, apparaissait comme le socle symbolique de l'aristocratie de l'ancienne Florence ${ }^{15}$. En mal de fondements théoriques susceptibles de justifier son intention de mettre définitivement fin aux idéaux des républicains, le nouveau régime développa à grand frais l'aristotélisme, compris comme une philosophie capable de fonder une politique pragmatique, d'inspiration monarchique - voire machiavélienne. On diligenta en particulier une série de traductions et de paraphrases en langue toscane des textes moraux, politiques et rhétoriques d'Aristote, toutes parues chez l'imprimeur officiel du grand duc, Lorenzo Torrentino, qui publia aussi nombre des leçons publiques données dans l'Académie Florentine ${ }^{16}$.

De cet Aristote toscan, traduit et paraphrasé, Bernardo Segni fut le principal ouvrier. Issu d'une famille anciennement alliée aux républicains, mais rallié au nouveau pouvoir, Segni était également membre de l'académie florentine. Il produisit en quelques années un petit corpus aristotélicien taillé aux besoins de la fragile légitimité de Cosme ${ }^{17}$. Si l'on en croit les formulations employées par ce traducteur dans la dédicace de son Trattato dei Governi, adressé à Cosme lui-même, Aristote aurait ainsi été partisan pragmatique d'une monarchie éclairée, en tous points semblable à celle mise en œuvre par $\operatorname{Cosme}^{18}$. De même, l'Éthique à Nicomaque est présentée par Segni à son dédicataire comme un texte susceptible de rendre ses sujets plus obéissants, tout en étant un traité où ce prince retrouvera l'image de son gouvernement vertueux ${ }^{19}$. Pour sa traduction en toscan de la 
Rhétorique et de la Poétique, Segni s'aida grandement du savant commentaire de Robortello sur la Poétique, paru seulement une année auparavant ${ }^{20}$. C'est que les Explicationes de Robortello, elles aussi publiées par Torrentino - dans un grand in-folio d'apparat, faisant état sur leur page de titre de quatre privilèges propres à leur assurer une diffusion internationale $e^{21}$ - étaient destinées à s'inscrire dans les mêmes types d'usages que ceux que Segni postulait pour ses traductions. Dans le cadre ses diverses obligations parauniversitaires, Robortello aurait d'ailleurs prononcé une partie de ce qui devint le proemium de son commentaire de la Poétique d'Aristote (p.1-5) devant l'académie florentine ${ }^{22}$.

9 Mais le texte de ces Explicationes est aussi un des éléments qui nous autorisent à soupçonner que Robortello n'était sans doute pas parfaitement à son aise avec les options politiques du gouvernement établi par Cosme. Or, en raison de l'éclatement politique des universités de la péninsule, qui dépendaient de pouvoirs locaux toujours en concurrence, l'opération de séduction que Robortello avait menée avec succès dans l'entourage du grand duc de Toscane, pouvait être répétée ailleurs, pour obtenir la bienveillance ou la protection d'autres institutions ou individus avec lesquels l'érudit se serait senti plus en accord sur le plan politique. La pluriactivité, imposée aux humanistes tout autant que construite par eux, avait ainsi, lorsqu'elle était pratiquée avec habileté, pour contrepartie de permettre aux érudits de jouer sur la diversité politique des différents lieux de savoirs. Alors même que Robortello semble à première vue ne songer, avec ses Explicationes, qu'à satisfaire l'entourage lettré de Cosme, certains indices, tant intratextuels qu'extratextuels, laissent à penser que son premier grand commentaire publié devait aussi servir, dans son esprit, à séduire le sénat vénitien, qui détenait le pouvoir de nomination en dernier ressort pour les principaux lieux de savoir de la Vénétie. Tout en promettant longue fidélité à Cosme dans la dédicace de ses Explicationes, Robortello aurait donc préparé dès 1548 (l'achevé d'imprimer de ses Explicationes date du mois d'octobre) son départ pour la Scuola di San Marco à Venise, soit pour le lieu de savoir chargé de former les futurs officiers de la chancellerie vénitienne. Le sénat lui confia dès le printemps 1549 la plus prestigieuse des deux chaires d'humanités grecques et latines de cette institution. Si la préméditation est en l'espèce vraisemblable c'est que, en acceptant ce poste, Robortello prenait la succession du célèbre Giambattista Egnazio. Or, contrairement à l'usage, Egnazio n'avait pas occupé sa chaire jusqu'à sa mort : il avait obtenu du sénat, après des demandes répétées, de pouvoir se retirer en considération de son âge avancé et de ses nombreuses années de bons et loyaux services. Sa succession avait fait naître des convoitises anticipées. Une telle configuration aurait même pu permettre à Egnazio de suggérer lui-même certains candidats aptes à prendre sa suite. Nul doute aussi que, pour triompher de ses rivaux, Robortello sut trouver du soutien aussi bien chez les érudits vénitiens que parmi les patriciens du sénat. Dans cette entreprise, son commentaire de la Poétique devait pouvoir être mobilisé comme faire-valoir, soit comme une manifestation de son savoir-faire philologique tout autant que politique. Mais publier un texte susceptible de convenir à l'entourage de Cosme-que la prudence commandait de ne pas froisser tant que rien n'était assuré à Venise -, tout en ne se fermant pas les portes d'une institution telle que la Scuola di San Marco demandait, pour le moins, une grande habileté énonciative. Alors que se développait à Florence une théorie de la nécessité, et même des vertus, du règne héréditaire d'un seul, le mythe politique vénitien était fondé sur une conception oligarchique d'un pouvoir destiné à être constamment partagé entre des patriciens que, en toute théorie, seuls leurs mérites et leurs vertus devaient conduire aux positions de pouvoir. Il était difficile de trouver, en 
Italie, deux mythologies politiques plus ouvertement contraires. Si Robortello voulait ménager ses patrons florentins, tout en attirant l'attention des nouveaux protecteurs qu'il convoitait, force lui était donc de développer des stratégies d'écriture et de publication cultivant l'ambivalence. Un usage prudent et mesuré de l'équivoque lui permit en l'espèce de rassurer l'entourage de cosme, tout en prétendant avec habileté aux bonnes grâces du sénat vénitien.

C'est l'analyse de ces stratégies de publication et d'écriture qui nous occupera, dans les pages qui suivent, à travers l'analyse d'un exemple. Le texte choisi est celui des réflexions que Francesco Robortello consacra, dans ses Explicationes de 1548, aux lignes 1449b 24 à 28 de la Poétique d'Aristote ${ }^{23}$ - soit le passage qui devint célèbre, en grande partie en raison de ce commentaire même, pour son évocation de la « catharsis » tragique. Cet exemple a été choisi d'abord pour ce qu'il manifeste des usages et enjeux de l'équivoque, à l'orée de l'époque moderne, au sein d'un discours d'érudition. Mais il est parallèlement scruté ici dans la perspective de cerner quelles furent, dans ce cas précis, les effets seconds de ces écritures équivoques sur la constitution en "doctrine ", au cours des XVI et XVII ${ }^{\mathrm{e}}$ siècles, des discours théoriques sur la poésie et la poétique. Cet exemple est convoqué enfin en tant que lieu textuel premier, mais aujourd'hui très largement oublié comme tel, d'un " problème » qui continue à fasciner tous ceux que la poétique préoccupe, soit celui de la «catharsis ». Le texte de Robortello sera donc analysé aussi comme l'un des nœuds théoriques originaires où furent rassemblés un ensemble d'éléments discursifs et doctrinaux qui continuent à hanter les discours sur la "purgation des passions». Il s'agira alors de réfléchir aux mécanismes par lesquels l'équivoque du texte de Robortello, faute d'avoir été abordée en tant que telle, continue à générer débats et malentendus, non seulement dans les écrits s'interrogeant sur le sens de la "catharsis », mais encore dans ceux qui, depuis le XVII ${ }^{e}$ siècle, prétendent éclaircir le texte d'Aristote (et ceux de ses innombrables commentateurs) en retraçant l'histoire de ce débat pluriséculaire.

\section{«Catharsis » : l'invention d'un problème philosophique}

11 Choisir, en 1548, de publier un grand commentaire de la Poétique d'Aristote était se saisir d'un texte qui n'avait encore donné lieu, dans l'Europe moderne, à aucune élucidation savante sous forme imprimée. Mais le sujet n'en était pas moins brûlant: ce texte connaissait depuis peu une grande popularité parmi les professeurs d'humanités du nord de la péninsule.

Le Moyen Âge n'avait le plus souvent connu l'existence de réflexions aristotéliciennes sur la poésie qu'à travers une traduction latine du Commentaire moyen qu'en avait donné Averroès $^{24}$. Certes, de rares manuscrits grecs de la Poétique avaient circulé en Europe depuis les $\mathrm{X}^{\mathrm{e}}-\mathrm{XI}^{\mathrm{e}}$ siècles ${ }^{25}$. Mais ce texte d'Aristote ne fut plus largement diffusé en Occident qu'à partir du Xve siècle, avec l'arrivée en Italie, puis en France, des manuscrits d'origine byzantine. Ange Politien posséda ainsi un manuscrit de la Poétique, qu'il annota avec soin, et que Robortello consulta, dans la bibliothèque des Médicis, pour préparer son propre commentaire ${ }^{26}$. Mais, malgré l'intérêt naissant de quelques humanistes avertis tels que Giorgio Valla, la Poétique ne suscita d'abord guère la curiosité des philosophes spécialisés dans le commentaire du corpus aristotélicien. Comme la Rhétorique, elle ne figura pas dans l'édition princeps du corpus, publiée par Alde Manuce entre 1495 et 1498, en dépit de l'existence d'une traduction latine du texte grec par Giorgio Valla parue dès $1498^{27}$. 
13 Les humanistes eux-mêmes ne s'emparèrent réellement du texte qu'après la diffusion, en 1536, d'une traduction latine de meilleure qualité, due à Alessandro de'Pazzi ${ }^{28}$. Bartholomeo Lombardi fut ainsi invité par l'académie padouane des Infiammati à prononcer en italien plusieurs leçons publiques sur la Poétique en 1541, en remplacement de celles que, sur le même sujet, Vincenzo Maggi n'avait pu donner l'été précédent ${ }^{29}$. Ces premiers commentaires, dans ce cadre para-universitaire, s'ébruitèrent immédiatement : de Florence, Cosimo Rucellai en réclama le texte à l'une des figures centrales de l'académie, Benedetto Varchi, par une lettre pressante ${ }^{30}$. Très vite, le sujet devint topique parmi les enseignants en humanités: Maggi, nommé à Ferrare en 1543, y enseigna la Poétique alors même que Robortello la paraphrasait à Pise ${ }^{31}$. D'un point de vue sociologique, cet intérêt soudain pour la Poétique (et la Rhétorique) d'Aristote s'explique probablement par le fait que ces textes permettaient aux humanistes d'anoblir leurs préoccupations courantes, centrées sur l'enseignement de la poésie latine et grecque et les grands textes de la tradition rhétorique, en les baignant d'un halo de philosophie. Qu'Aristote ait traité de poésie et de rhétorique - c'est-à-dire de sujets généralement tenus pour mineurs par les métaphysiciens ou les spécialistes de philosophie naturelle - était assez pour valoriser ces savoirs, et rendre plus considérables ceux qui les enseignaient.

\section{La Poétique au service de Cosme de Médicis ?}

De telles considérations furent sans doute aussi à l'origine du choix de Robortello lorsqu'il décida de faire de la Poétique le sujet du premier commentaire d'envergure publié sous son nom. L'opportunité politique, qui exigeait des humanistes florentins un Aristote toscan, destiné à appuyer le pouvoir médicéen, ne fut cependant pas moins déterminante. L'auteur des Explicationes ne manque en tout cas pas de présenter son livre comme un objet destiné à servir au mieux son prince. Pour ce faire, il ne se contente pas, dans son épître dédicatoire, d'insister sur son ardeur au travail et sur son très grand désir de demeurer dans la clientèle de Cosme ${ }^{32}$. Il s'efforce aussi d'expliquer pourquoi l'objet qu'il a choisi est parmi les plus aptes à servir les besoins politiques de son patron. Aristote, affirme-t-il, après avoir très habilement traité de toutes les sciences de la nature (eam philosophice partem, quœe naturce vim, et rerum causas scrutatur), s'intéressa aux arts du discours, à l'exemple d'Isocrate qui s'y consacrait alors exclusivement. Et, la philosophie étant la mère de tous les savoirs, Aristote, qui analysa et classa toutes les choses

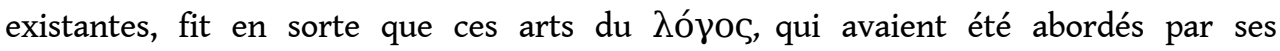
prédécesseurs de manière confuse et désordonnée, se trouvent décrits selon une rationalité sûre et systématique (arteis eas, quœ antea ab aliis confuse, ac perturbate tractatce fuerunt, ad certam quandam rationem, ac seriem redigere). Si Robortello insiste sur le soin avec lequel Aristote aborda ces domaines, c'est avant tout pour souligner la valeur de la rhétorique et de la poétique en tant que " arts " à part entière, que les aristotéliciens de son temps, jugeant ces précieux savoirs tout à faits triviaux, auraient, selon lui, une fâcheuse tendance à négliger :

[...] beaucoup a déjà été écrit sur les textes d'Aristote, qui a traité de tous les types de philosophie, mais concernant la rhétorique et la poétique, qui sont deux arts très importants et très excellents, ah! Dieu du ciel, avec quelle dextérité, avec quelle netteté, quelle érudition et quel savoir ce philosophe en a traité dans les écrits qu'il nous a laissés ; Aristote avait pour habitude de désigner ce type de réflexions par les

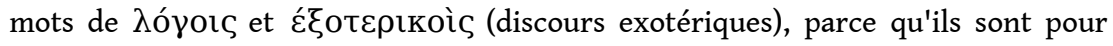


ainsi dire plus connus de tout un chacun et plus répandus parmi nous, et parce qu'ils sont plus souvent que les autres mobilisés par les hommes dans l'espace public et en pleine lumière ; c'est sans doute la raison pour laquelle je ne vois pas qu'on lise beaucoup, chez ceux qui de nos jours se donnent le titre de philosophes aristotéliciens, les livres d'Aristote qui traitent de la rhétorique et de la poétique, ni qu'on en disserte abondamment; et je ne crois vraiment pas que ce soit en raison de leur complexité (car nos philosophes en élucident de bien plus complexes), je crois au contraire que, soit parce qu'ils tiennent ces textes pour trop triviaux pour qu'ils soient désireux d'y adosser leur œuvre, soit parce qu'ils se satisfont de la connaissance de quelques réalités - connaissance qui en vérité est bien peu de choses -, ces gens négligent les arts qui ont trait à une ornementation plus soignée, et à une pompe en quelque sorte théâtrale et publique ${ }^{33}$.

Robortello accuse les philosophes scolastiques de mépriser indûment la rhétorique et la poétique, deux savoirs pourtant accessibles et fort nécessaires au commerce des hommes. De telles réflexions témoignent, ici encore, de la concurrence, dans le cadre des universités italiennes, entre les professeurs d'humanités, dont l'enseignement était considéré comme propédeutique (et les chaires demeureraient moins bien rémunérées) et les professeurs de philosophie naturelle et de métaphysique, dont les cours, fondés sur le commentaire des textes physiques et métaphysiques d'Aristote, constituaient le couronnement du curriculum dans les facultés des arts. Mais elles sont aussi le signe d'une autre conception des rapports entre "sciences » et " arts ", le savoir des humanités étant présenté comme plus susceptible d'être mis au service d'une cité-c'est-à-dire, généralement, au service d'un pouvoir. Ces "arts» que les scolastiques jugent moins substantiels (leviora) que les savoirs des obscurs traités qu'il commentent - et donc moins susceptibles de leur acquérir gloire et renom-sont d'après Robortello des «arts » essentiels à la vie d'une communauté politique : ils recouvrent tout ce qui a trait aux ornements raffinés, à la splendeur, voire à une pompe toute spectaculaire, soit plus largement tout ce qui permet de projeter une image de son point de vue ou de soi-même dans l'espace public. Robortello définit ainsi la rhétorique et la poétique comme des "arts" de la représentation-et ce faisant, insinue qu'ils seraient tout à fait indispensables aux gouvernants. C'est pourquoi, s'il est beau de s'interroger sur le mouvement des sphères ou la nature des éléments, "il est tout à fait honteux ", selon Robortello, « de tout ignorer de l'antiquité et de ne pas toucher aux arts qui règnent au milieu des assemblées, dominent les esprits des hommes, et pénètrent jusqu'à leurs convictions intimes, [et qui permettent que] des volontés diverses, des goûts différents et des personnalités dissemblables ne soient tempérées par rien d'autre que le raisonnement, et, par là, conduites où l'on voudra ${ }^{34}$.» D'où il ressort nettement que, comme l'ensemble de ses contemporains, Robortello ne semble concevoir ici la poétique que comme un savoir inféodé non seulement à la rhétorique, mais encore à la philosophie morale et politique ${ }^{35}$. Confrontées aux spéculations des physiciens scolastiques, rhétorique et poétique sont ici vantées comme des savoirs socialisés, susceptibles d'être mis au service des hommes, et, plus spécifiquement, du prince.

\section{Éclaircir ou commenter : usages équivoques de l'érudition}

16 À la lecture d'une telle dédicace, la tentation serait grande de penser que Robortello ne pouvait aller, dans son interprétation de la Poétique d'Aristote, que dans le sens d'une instrumentalisation morale et politique du théâtre, et, plus largement, des arts du discours. On découvre pourtant à la lecture de certains des passages clés de son 
commentaire que rien n'est moins vrai: dans l'analyse de certains éléments du texte aristotélicien, Robortello joua au contraire de toutes les possibilités que lui offraient son érudition, et, plus largement, les manières de faire de la tradition doxographique, pour créer dissonances et perplexités, prenant discrètement ses distances avec toute idée d'instrumentalisation politique de la poésie. C'est en particulier le cas de son commentaire des lignes $1449 \mathrm{~b} 24$ à 28 , dont Robortello inventa ou du moins réinventa, au sens rhétorique du terme ${ }^{36}$, la complexité, en combinant une présentation ambivalente de ses propres réflexions avec la mobilisation délibérée de propos contradictoires tirés d'autorités judicieusement choisies à cette fin.

\section{De la philologie à la philosophie}

17 Car la "purgation des passions", devenue un lieu commun de la philosophie morale après 1570 , n'avait pas un statut de topique avant que Robortello ne la (re)constitue en quœstio par son commentaire de la Poétique. Pour autant qu'on puisse en juger par les manuscrits qui nous sont parvenus, la « catharsis » ne constituait d'ailleurs probablement pas une notion problématique aux yeux d'Aristote, qui utilise ce terme sans prendre la peine de souligner qu'il soulèverait des problèmes philosophiques spécifiques ${ }^{37}$. La " catharsis » semble même avoir indifféré les penseurs médiévaux : le Commentaire Moyen d'Averroès, largement diffusé entre 1300 et 1500 , ne mentionnait pas cette forme particulière de "purification " par lequel Aristote semblerait avoir voulu justifier des usages moraux et politiques de la tragédie. Cette omission peut s'expliquer soit par la médiocrité de la traduction (probablement) syriaque de l'hypothétique texte grec à partir duquel ce commentateur aurait œuvré, soit par la logique propre du commentaire qu'Averroès produisit. Attribuant, comme l'ensemble des penseurs arabes médiévaux, une visée logique, morale et sociale, à la rhétorique comme à la poésie ${ }^{38}$, Averroès n'avait sans doute nul besoin de mettre en avant un élément relativement mineur (du moins dans les manuscrits dont nous disposons) du texte d'Aristote.

Les commentaires de Maggi et de Lombardi, dont Robortello aurait pu avoir connaissance par ouï-dire ou sous une forme manuscrite ${ }^{39}$, sont susceptibles d'avoir pu attirer son attention sur la «catharsis». Mais, à juger par la version imprimée de leurs Communes explanationes (1550), cette notion ne soulevait guère, aux yeux de Maggi et de Lombardi, de problèmes herméneutiques majeurs. Au cours de l'explanatio que le livre publié par Maggi attribue aux deux auteurs, ce passage est simplement désigné comme celui où Aristote indiquerait le plus clairement la « fin» de la poésie, laquelle serait de purger chacun de passions telles que le désir et la colère ${ }^{40}$. Dans les annotationes que Maggi ajouta à ce commun commentaire, seul est précisé qu'Aristote ne suggère pas que la terreur et la pitié délivreraient les spectateurs de "ces mêmes passions »-ce qui, d'après Maggi, serait absurde puisque alors la cause se détruirait elle-même, sans compter que la pitié et la terreur sont essentielles à la charité comme à l'obéissance -, mais bien seulement de passions « semblables", telles que l'avarice ou la luxure, qui peuvent alors laisser place à des vertus ${ }^{41}$. En spécifiant de manière claire les référents auxquels renverraient d'après

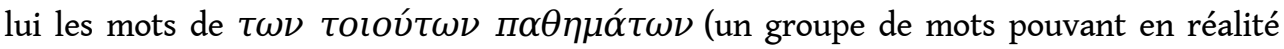
signifier « de telles passions » tout aussi bien que « ces mêmes passions »), rendus en latin par perturbationes huiusmodi, Maggi ne faisait en réalité qu'expliciter la pensée de Lombardi. Il proposait une solution philologique nette et tranchée - quoique âprement discutée depuis lors - à un problème (l'absence d'un réfèrent clair pour le démonstratif 
$\tau o เ o u ́ \tau \omega v)$, qui tient essentiellement à une ambivalence du texte-source. En guise d'explication du terme " catharsis », Maggi citait ensuite le passage de la Politique (1342a 1 à 15) qui décrit sommairement les modalités de cette purgation. Celle-ci se trouvait ainsi implicitement placée dans un contexte politique, mais les enjeux d'une telle contextualisation n'étaient pas mis en évidence. Car le passage de la Politique n'était mobilisé dans le texte de Maggi que comme support d'une philologie qui en reste aux problèmes sémantiques : le texte de la Politique est présenté comme donnant le sens d'un mot dont, sans lui, les référents resteraient obscurs. Le recoupement intertextuel étant l'une des principales techniques philologiques, il était d'ailleurs difficile de ne pas faire apparaître le texte de la Politique dans un commentaire de la notion de "catharsis " tragique, puisque Aristote lui-même renvoyait à sa Poétique en ce lieu de la Politique.

Or, quoiqu'il soit probable que Robortello ait eu vent des leçons professées par Maggi avant $1548^{42}$, son propre texte fonctionne sur un mode très différent : dans le cadre de son commentaire de ce passage, les problèmes linguistiques intéressent apparemment peu l'auteur des Explicationes, qui donne, dans son commentaire, la première place au questionnement des enjeux moraux, philosophiques et politiques de la notion de « catharsis ». Pour ce faire, il fait appel à un philosophe de l'Antiquité qu'il fut le premier philologue moderne à (re)mobiliser cette notion dans le cadre d'une lecture de la Poétique d'Aristote : Proclus.

\section{L'interprétation est un « piège »}

20 À la différence des textes publiés par Maggi après la disparition de Lombardi, le commentaire de Robortello se divise en deux parties d'égale longueur mais de nature sensiblement différente. La première se cantonne à une contextualisation de la particule concernée dans l'ensemble du corpus aristotélicien. La seconde contient une discussion de l'utilité morale et politique de la tragédie, construite à partir de la convocation doxographique de trois autorités antiques parmi lesquels le commentaire de Proclus sur la République de Platon se voit attribuer la place d'honneur. Ce dispositif construit de l'ambivalence à un double niveau. L'équivoque jaillit d'abord de la juxtaposition de ces deux parcours interprétatifs - l'un philologique, l'autre philosophique - aux implications contraires, entre lesquelles le commentateur s'abstient de trancher explicitement. L'incertitude est portée à son comble, lorsque, dans la discussion de la quœstio de l'utilité morale et politique de la tragédie, le commentateur se refuse à dire laquelle des autorités mobilisées par lui (soit Proclus, Sextus Empiricus, et Plutarque) emporte sa préférence. Le lecteur s'interroge tout autant sur la signification du conflit latent entre les deux moments de ce commentaire que sur la mobilisation, dans le second moment du texte, d'autorités auxquelles Robortello attribue des postions parfois non moins clivées - et par conséquent non moins potentiellement équivoques-que la sienne. Organisant une perpétuelle hésitation entre éclaircissement philologique et interprétation philosophique, le commentaire de Robortello se révèle ainsi doublement un " piège ${ }^{43}$ ".

21 La première partie du commentaire (p. 52-53) est donc constituée par une analyse mot à mot de la particule, que Robortello prolonge par une lecture de la notion de " catharsis » dans la pensée d'Aristote, en s'appuyant sur un rapprochement avec deux passages du livre VIII de la Politique, l'un tiré du chapitre 5 (1340a 12-22) et l'autre du chapitre 7 (1341b 36 à 1342a 15). Dans la linéarité de cette première approche, l'explication de la notion de purgatio perturbationum occupe en réalité plus de la moitié du propos du commentateur 
(soit le bas de la p. 52 et toute la p. 53) : le texte de Robortello présente implicitement la purgatio comme le nœud gordien de la définition qu'Aristote donnerait de la tragédie. La conclusion de ce premier moment en témoigne :

Si quelqu'un demande quelle est la doctrine d'Aristote sur la tragédie, je réponds qu'il [le Philosophe] estime que ces déclamations et spectacles purgent de deux passions, à savoir la crainte et la pitié. Lorsque les hommes assistent à ces déclamations, ils entendant et ils voient des personnes parlant et faisant des choses qui se rapprochent beaucoup de la vérité, ils s'habituent à souffrir, à craindre et à avoir pitié, de telle sorte que si quelque chose survient dans la vraie vie, ils souffrent moins et ont moins peur; il est en outre nécessaire que celui qui n'a jamais souffert de quelque malheur souffre de manière exagérée, si quelque chose d'adverse lui arrive sans qu'il s'y attende. Ajoutons que souvent les hommes souffrent et sont pleins de crainte devant des choses de peu d'importance; au contraire les poètes dans les déclamations de leurs tragédies leur offrent à voir des personnes et des choses extrêmement dignes de commisération et que quiconque, même sage, redoute à juste titre et au plus haut point; ainsi les hommes apprennent quelles sont les choses qui font légitimement naître de la commisération et de la douleur, et quelles sont celles qui font à juste titre surgir la peur. Enfin, les auditeurs et les spectateurs comprennent cette utilité, qui est maximale : en effet puisque le sort commun des hommes est qu'il n'y a personne qui ne soit sujet aux malheurs, les hommes supporteront plus facilement que quelque chose d'adverse se produise et tiendront bien ferme [dans leur malheur] par une consolation des plus solides, s'ils se souviennent que la même chose est arrivée à d'autres. ${ }^{44}$

Précisant clairement qu'il ne parle ici qu'en tant qu'interprète de la « doctrine » (sententia ) d'Aristote sur la tragédie, Robortello affirme d'abord qu'il s'agit bien, dans le texte du philosophe, de purger les spectateurs de la pitié et de la crainte, en les accoutumant aux peines que toute vie humaine comporte par le spectacle des souffrances des personnages du théâtre. Le commentateur adopte donc une lecture du texte d'Aristote qui est contraire à celle défendue par Lombardi puis par Maggi. Mais surtout, à la différence de ce dernier commentateur, Robortello désigne clairement la conséquence des recoupements avec la Politique d'Aristote qu'il vient de mettre en évidence : si la théorie de l'accoutumance aux souffrances par la contemplation d'actions tragiques est acceptée comme fondement de la réflexion, il faudra en conclure à l'« utilité » morale et politique de la tragédie, soit à sa capacité à rendre les hommes plus capables de supporter leurs maux. Pour illustrer cette thèse, mais non pour l'appuyer, Robortello cite en grec un passage des Deipnosophistes d'Athénée (III ${ }^{\mathrm{e}}$ siècle après J.-C.) dans lequel le poète comique Timoclès - un contemporain d'Aristote - est cité comme ayant décrit, dans une pièce intitulée Les Dionysies, les multiples manières par lesquelles la tragédie peut être utile ( $\chi \rho \eta ́ \sigma \tau \mu \varsigma)$ aux hommes ${ }^{45}$. Les vers cités par Athénée et repris in extenso par Robortello semblent d'abord défendre l'idée que les maux des héros tragiques, tels qu'ils sont représentés dans les tragédies, montrent à ceux qui souffrent des douleurs bien plus terribles que les leurs et encouragent ainsi tous les spectateurs à accepter leurs malheurs avec plus de patience. Les philologues s'accordent néanmoins actuellement pour dire que le texte de Timoclès est ironique et que sa tragédie des Dionysies raille en cet endroit les positions d'Aristote sur la tragédie ${ }^{46}$. Une telle position d'énonciation n'avait sans doute pas échappé à Robortello, qui fait ainsi, selon toute vraisemblance, un usage équivoque du texte cité par Athénée. Mobilisés par l'humaniste italien sans contextualisation ni commentaire, les vers de Timoclès pourraient d'abord paraître se borner à résumer les positions attribuées à Aristote (selon lesquelles la tragédie rendrait les hommes plus vertueux et peut-être même plus aisément gouvernables). Mais, un lecteur qui connaît les 
manières de faire des doxographes grecs de la période tardive, perçoit simultanément que le discours de Timoclès, rapporté par Athénée et repris par Robortello, permet en réalité au commentateur italien de mettre subtilement à distance la conception aristotélicienne de la tragédie.

Une lecture de la "doctrine» d'Aristote concernant la tragédie a néanmoins été proposée : la tâche de l'élucidateur, telle que la comprenaient par exemple Lombardi et Maggi, pourrait donc être tenue pour accomplie. Cependant, autre signe que le commentateur ne se trouve sans doute pas en plein accord avec le penseur qu'il commente, l'éclaircissement philologico-contextuel ne constitue dans le texte de Robortello qu'une première étape. Son texte se poursuit par une mise en débat de la pensée d'Aristote, où la «catharsis » est construite en quoestio. Mais au lieu de prendre position dans le débat qu'il agite (dans un moment rhétorique que la scolastique désignait par le verbe respondeo), le commentateur laisse sa question sans réponse, construisant ainsi la «catharsis » en problème irrésolu, voire insoluble. Ceci est d'autant plus vrai que la discussion n'est pas réellement assumée par le commentateur : elle se fait par le biais de la mobilisation doxographique de trois autorités parmi lesquelles Robortello ne tranche pas. Faisant néanmoins état d'opinions contraires à celles qu'il a attribuées à Aristote, le commentateur met indirectement en cause l'utilité politique de la poétique et de la rhétorique, pourtant affirmée par lui-même dans sa dédicace à Cosme.

Le premier texte convoqué dans ce second moment est le commentaire de Proclus sur La République de Platon ${ }^{47}$. Car c'est en réalité en allant chercher ce commentateur néoplatonicien de Platon que Robortello réinvente la qucestio de la «catharsis » et l'introduit sur la scène intellectuelle du Cinquecento italien. Le commentateur avance néanmoins avec une extrême prudence puisque, tout en louant la grande érudition de ce philosophe, il ne le présente pas explicitement comme un disciple de Platon, se contenant d'expliquer que Proclus remarque, en abordant les quostiones poetices de son commentaire, une dissension entre Aristote et Platon sur ce point, avant de s'efforcer de réfuter les opinions d'Aristote sur la poésie ${ }^{48}$. De même, c'est en tronquant une citation du texte grec de Proclus, où celui-ci souligne que Platon souhaitait voir les poètes bannis de sa république, que Robortello indique discrètement aux hellénistes que le commentaire du philosophe platonicien se centre sur le problème des fonctions sociales et politiques de la poésie ${ }^{49}$. Lorsqu'il choisit de rendre compte du raisonnement de Proclus, le commentateur de la Poétique choisit néanmoins de donner un résumé en latin de cette doctrine, s'appuyant même sur une traduction mot à mot de certaines des expressions employées par le philosophe platonicien ${ }^{50}$. Tel que Robortello en rend compte, le propos de Proclus serait que toute imitation imitant des choses variées - c'està-dire bonnes aussi bien que mauvaises -, les produits d'une telle activité sont des images déréglées, qui s'insinuent dans les âmes des spectateurs et les détournent du chemin de la vertu. La tragédie et la comédie sont donc à fuir, en tant qu'imitations de choses bigarrées, car ces imitations remplissent la vie des maux qui proviennent de mœurs mêlées et impures. À la fois très proche du texte de Proclus, et plus synthétique et même parfois plus catégorique que lui, le type de glose pratiqué par Robortello ne permet plus guère de savoir s'il parle exclusivement au nom de Proclus ou aussi en son propre nom. Le commentateur semble parfois reprendre implicitement à son compte les propos qu'il rapporte-comme le marquent des expression telles que quod vocamus utilisées pour signaler les termes que Robortello emprunte à Proclus -, mais son lecteur ne peut en 
réalité jamais être certain qu'il fasse pleinement siens par cet usage de la première personne du pluriel.

Lorsqu'il en vient alors au commentaire des lignes 50.8 à 29 du texte de Proclus ${ }^{51}$, qui semblent constituer pour lui le cœur de l'argumentation du philosophe platonicien, Robortello commence par citer en grec le texte-source, avant de le gloser dans les deux langues, en réagençant les arguments de Proclus selon ses propres besoins. En philologue confirmé, Robortello perçoit que les objections que Proclus fait à la doctrine aristotélicienne de la tragédie reposent avant tout sur une opposition entre le terme de

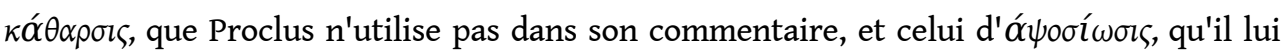
substitue. Ce second terme fait allusion à une purification (et même "sanctification ") de l'âme, semblable à celle donnée au cours des mystères d'Eleusis ${ }^{52}$. Proclus l'oppose implicitement à la purification toute charnelle et bassement émotionnelle que serait la кó $\theta \alpha \rho \sigma \iota \varsigma$ aristotélicienne. L'importance de cette substitution est soulignée par Robortello, qui alerte son lecteur avec insistance, soulignant que «ce mot [d'ó $\psi$ ooí $\omega \sigma \iota \varsigma]$ est à noter, car là se trouvent les éléments qui combattent la doctrine d'Aristote » (notanda vero hoec verba diligenter, nam ipsa sunt, quce oppugnant Aristotelis sententiam). Si, en effet la comédie et la tragédie ne purifient pas l'âme (neque utile sunt ad purgationem,) mais excitent au contraire les émotions les moins nobles des hommes - telles que le rire et les larmes -, elles ne sauraient avoir d'utilité politique. Cité en grec par Robortello, Proclus concède que le rôle du politique est bien d'imaginer des moyens pour dégorger les passions de son peuple, mais insiste sur le fait que le théâtre ne saurait servir à accomplir cette tâche. Paraphrasant Proclus de telle sorte que l'on ne sache plus si la doctrine qu'il présente est celle de Platon, celle de Proclus seulement, ou bien une position à laquelle lui-même adhère, Robortello conclut alors de la manière suivante :

Pour nous résumer, disons que deux raisons ont poussé Platon à rejeter les

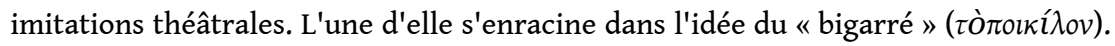
L'autre se rattache à l'idée que ces imitations émeuvent les passions de manière très excessive. Chacun doit en effet contenir de manière virile les affections de son âme. Ces dernières, que nous avons souvent nommées, on comprend qu'elles

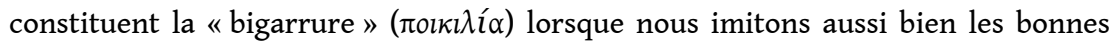
choses que les mauvaises, soit la continence et l'incontinence tout à la fois. Car Platon n'approuve pas de cette bigarrure, puisque si la poésie vise à l'utilité et à l'instruction des hommes, il n'est pas nécessaire d'imiter quiconque, hormis les hommes bons et sages. Néanmoins (comme Proclus le dit dans sa première quoestio), les hommes prennent toujours naturellement plaisir aux imitations, et, de ce fait, nous sommes tous « amateurs de fables » ( $\psi \imath \lambda o ́ p v \theta o l)$, ce qui fait que, [comme l'écrit Proclus au lieu sus-mentionné] si nous sommes habitués à vivre «avec des imitations bigarrées et variées, nous nous assimilons à elles en raison de notre sympathie, ainsi nous devons bigarrés quant aux mœurs et nous nous réjouissons de choses bigarrées ». Or cette imitation bigarrée est très agréable mais elle n'est pas utile à l'éducation des hommes. ${ }^{53}$

On voit que cette conclusion n'est pas équivoque dans son interprétation de Platon au travers de Proclus: seul le statut de son énonciation reste problématique, puisqu'elle laisse le lecteur dans le doute quand à la question de savoir ce qu'en pense Robortello luimême : nul ne peut dire si la dernière phrase du paragraphe est à comprendre comme l'explication de la pensée de Proclus ou s'il faut aussi comprendre que son commentateur italien la reprend à son compte.

Mais en quoi révéler son accord avec Proclus sur l'interprétation philosophique d'une notion telle que la "catharsis" pouvait-il se révéler risqué pour un homme dans la position de Francesco Robortello? Une conviction de ce type semble ne menacer aucun 
dogme, et ne vise, du moins en apparence, aucune autorité. Manifester son accord avec le commentaire de Proclus pouvait néanmoins s'avérer imprudent pour au moins deux raisons.

La première tient au texte même de Proclus. Car si les passages cités en grec ou paraphrasés en latin par Robortello semblent en effet relativement anodins, certains des passages qu'il ne cite pas - mais qu'un helléniste pouvait aisément retrouver puisqu'ils figurent, dans la linéarité du texte, entre les extraits dont Robortello se fait l'écho ${ }^{54}$ - l'étaient sans doute beaucoup moins. Ainsi, par exemple, le passage dans lequel Proclus concède que si la poésie dramatique peut avoir une utilité politique, ce ne sera que dans le cadre des régimes qui s'éloignent le plus de la république idéale :

[...] aussi pourrions nous trouver que l'art qui imite la bigarrure des mœurs a peutêtre quelque utilité pour certaines gens, ceux pour lesquels le non-bigarré est plus nuisible que le bigarré. C'est pourquoi cette poétique [plus exactement: cette poésie] est profitable au régime tyrannique absolu, puisqu'elle ne laisse pas le tyran se complaire au seul genre de vie qui est le pire, mais qu'elle y ajoute le comportement dérivé de l'imitation de toutes les sortes de mœurs, car elle embrasse à la fois les pratiques les meilleures et les pires. Et en effet, comme il semble, de même que le bigarré est nuisible au régime royal et divin, de même est-il utile au régime le plus inférieur et tyrannique. Il est sûr du moins que le simple est de deux sortes, ou supérieur au bigarré, ou inférieur. Le supérieur ne saurait qu'être endommagé en s'adjoignant le bigarré, il deviendrait pire, comme s'étant infecté du pire. L'inférieur en revanche ne saurait trouver que du profit à s'adjoindre le bigarré, il deviendrait meilleur, comme bénéficiant du meilleur. ${ }^{55}$

On imagine aisément que citer un tel passage dans un ouvrage adressé à Cosme $1^{\text {er }}$ - que les défenseurs de la république s'étaient depuis longtemps empressés de qualifier de « tyran »- aurait été un geste susceptible de déplaire. Suggérer à Cosme - qui faisait tant d'efforts pour apparaitre en généreux patron des lettres, et prêtait attention à l'agencement de tout portrait, fête curiale ou monument destiné à véhiculer de lui une image de prince magnanime ${ }^{56}$ - que son goût pour ces panachés de bien et de mal que seraient les imitations révélait en réalité la nature corrompue du régime qu'il imposait à Florence, et même la vilenie de ses propres mœurs, aurait été pour le moins maladroit. Dans une telle perspective, les arts théâtraux - mais peut-être aussi l'ensemble de la rhétorique et de la poétique que la dédicace à Cosme s'efforçait d'anoblir - pourraient apparaitre comme de vulgaires cache-misères destinés, en désespoir de cause, à instiller quelque parcelle de vertu dans les cœurs corrompus du tyran et de ses sujets, ou pire, à voiler la tyrannie sous le faste de sa propre représentation. On comprend la prudence de Robortello dans son maniement du texte de Proclus. Sans nécessairement tenir cosme pour un tyran, il pouvait chercher à manifester quelque distance vis-à-vis du régime médicéen, tant politiquement qu'intellectuellement. Mais, s'il voulait maintenir sa position à Pise et dans l'entourage de Cosme, il était crucial que cette distance, si elle devenait perceptible, restât impossible à assigner.

Plus généralement, faire apparaittre clairement son accord avec la position de Proclus aurait été publier son (néo) platonisme, alors même que Platon n'était guère à l'honneur dans le contexte intellectuel et politique florentin des années 1540-1550. Et, bien que son commentaire de la Poétique porte effectivement de nombreuses marques de convictions platoniciennes, ou du moins d'un désir d'accorder les positions de Platon et d'Aristote sur la poésie, Robortello paraît avoir déployé tous ses efforts, dans son commentaire de la " catharsis ", pour que ses préférences n'apparaissent pas au grand jour. Une description du platonisme latent des Explicationes excéderait le cadre de ce travail. Il est perceptible 
notamment dans l'insistance que le commentateur, s'inspirant là encore de Proclus, met à penser la poésie comme mensonge vrai (in poeticis mendaciis principia falsa pro veris assumuntur, op. cit, dès la p. 2), soit dans son effort pour la décrire comme une énonciation des plus fallacieuses, mais néanmoins susceptible de faire apercevoir la vérité (et même le divin) mieux que tout autre type de discour ${ }^{57}$. Dans son commentaire sur la notion de "catharsis », Robortello tient cependant à maintenir cachée sa préférence pour la position (néo)platonicienne. En témoigne la manière dont s'achèvent les réflexions qu'il consacre à la particule d'Aristote. Car, sans donner explicitement son sentiment sur les réflexions de Proclus, Robortello mobilise immédiatement à leur suite deux autorités, dont la première ne fait qu'augmenter la perplexité de son lecteur, tandis que la seconde ne pouvait, mais seulement chez un lecteur averti, que faire davantage pencher la balance en faveur de Platon.

29 Apparait d'abord l'autorité qui invite par excellence à la suspension du jugement puisqu'il s'agit de la figure centrale du scepticisme: Sextus Empiricus. Robortello mobilise deux passages contradictoires des réflexions que, dans l'ouvrage connu sous le titre de Contre les savants ou Contre les professeurs, Sextus consacrait à montrer que les jugements des grammairiens sur la poésie étaient irréconciliables. Se référant aux positions des Epicuriens, Sextus écrit d'une part que la poésie est «inutile» et même «nuisible à la vie ", puisqu'elle incite chacun à se laisser aller encore davantage à ses passions (Contre les professeurs, I, 298). Mais, relève Robortello, Sextus mentionne aussi la position adverse, selon laquelle la poésie fournit de «nombreux points d'appuis [pour s'élancer vers] la sagesse ", ce qui s'expliquerait selon Sextus par le fait que les énoncés gnomiques de la poésie sont en réalité à l'origine de la philosophie, comme le montre l'habitude qu'ont encore les philosophes de ponctuer leurs injonctions de vers pris dans les poètes (Contre les professeurs, I, 271). Là, encore Robortello ne prend pas parti, se contentant, tout comme Sextus, de soumettre à son lecteur cette alternative construite pour apparaître comme tout à fait indécidable.

Pour mettre un comble à la perplexité, Robortello s'en réfère, pour finir, à une dernière autorité : "Voilà, écrit-il narquoisement, ce qui a été dit dans les deux camps par les anciens philosophes. Décider de ce qu'il faut penser de ces choses ne sera pas difficile, notamment parce que Plutarque a doctement et sagement jugé de quelle poésie, dans quelle mesure et quand, elle doit ou ne doit pas être écoutée ${ }^{58}$. » Ainsi le lecteur est-il prié d'aller chercher dans les considérations du Comment écouter les poètes de Plutarque ce que Robortello se refuse apparemment à lui dire. Quoique que bientôt supplanté dans son statut d'autorité par la Poétique d'Aristote et l'Art poétique d'Horace (deux textes que le commentaire de Robortello contribua en réalité grandement à établir dans cette fonction référentielle ${ }^{59}$ ), ce texte de Plutarque était alors une des sources majeures de toute réflexion sur la poésie ${ }^{60}$. Il traitait la question de savoir si la poésie pouvait légitimement prétendre faire partie de l'éducation morale des adolescents. Plutarque répondait lui aussi de manière doxographique et balancée à ce dilemme, puisque tout en semblant persuadé qu'il ne serait pas avantageux d'interdire la poésie aux adolescents (15a), il énonçait néanmoins dans la première partie de son dialogue tout ce qui rendrait les poètes dangereux (16a-28d), avant de donner, dans la seconde partie de son texte, toutes sortes de conseils de lecture pour pouvoir malgré tout tirer un profit moral de leurs œuvres (28e-37a). À la seule lecture de ce texte, l'alternative construite par Robortello semblerait donc demeurer ouverte. Mais Plutarque est immédiatement identifiable - du moins pour un lecteur disposant d'une formation philosophique --, comme un disciple de 
Platon, jusque dans ce dialogue de jeunesse qui prône un usage modéré de la poésie, tout en cantonnant son utilité au domaine de l'éthique ${ }^{61}$. C'est dire que, par l'appartenance de ce texte à la constellation du platonisme, tout autant que par l'absence, en son sein, de toute réflexion sur l'utilité spécifiquement politique de la poésie, le dialogue de Plutarque semblerait peu susceptible de faire pencher la balance en faveur d'Aristote. En le mobilisant en dernier ressort, par une phrase sibylline, et non dénuée d'ironie, Robortello permet à ses lecteurs les plus érudits de supputer ses propres positions, tout en maintenant jusqu'au bout l'équivoque, puisque, si l'on s'en tient au seul texte du dialogue, la position de Plutarque pourra toujours être tenue elle-même pour ambivalente.

\section{Une énonciation flottante, figée dans l'équivoque}

31 À la mise en scène doxographique d'un conflit irrésolu d'autorités s'ajoutent donc les ambiguïtés d'une énonciation cherchant par tous les moyens à paraître inassignable. Robortello aurait pu user d'un procédé souvent mobilisé par les érudits, qui prenaient soin de faire figurer tout ce qui flatterait leur patron dans la dédicace, en s'octroyant plus de liberté dans le corps du texte ${ }^{62}$. Le mécène ne lisant le plus souvent pas le latin, on pouvait légitimement penser que sa curiosité, si curiosité il avait, s'arrêterait aux textes liminaires. Mais Robortello n'était apparemment pas sans savoir que, si Cosme ignorait effectivement le latin, il usait au besoin des hommes de son entourage lettré pour déchiffrer et évaluer les textes érudits. Campana avait précédemment joué ce rôle auprès de lui et d'autres l'avaient remplacé dans l'entourage du prince, ou étaient en passe de le faire. C'est sans doute une des raisons pour lesquelles Robortello juge bon d'insérer dans le texte même de son commentaire, des éloges de Campana et des princes de la famille des Médicis (Alexandre puis Cosme) qui l'avaient protégé ${ }^{63}$. S'il pouvait penser que le corps du texte serait lui aussi scruté, force était donc, pour faire entendre quelque désaccord ou dissonance, de trouver un procédé plus subtil que celui qui consistait à camoufler les audaces du corps du texte sous les flatteries d'une épître dédicatoire.

À cette fin, Robortello, associant toujours philologie et philosophie, subvertit, dans son commentaire de la «catharsis », les «manières de faire ${ }^{64}$ » de l'érudition en mêlant les usages énonciatifs de la lectio oralisée et avec ceux du commentaire destiné à l'impression. Il produit ainsi une parole flottante, parce que détachée de tout contexte d'énonciation clairement identifiable. Comme celui publié par Pier Vettori en 1560, mais de manière plus prononcée encore, le commentaire de Robortello est un commentaire d'apparat, intégralement linéaire et rédigé avec le plus grand soin. Cependant, même si le texte cherche constamment à faire naître une impression d'oralité par la récurrence de locutions telles que quod ante fuit probatum (qui signale une répétition) ou verum de his hactenus (qui laisse croire que le commentateur se serait involontairement laissé emporter à quelque digression), les cours d'humanités délivrés dans les universités du Cinquecento ne fonctionnaient pas sur le modèle du commentaire linéaire in extenso adopté par l'ouvrage. Les notes préparatoires à un cours qui ont pu survivre de cette époque sont généralement d'une présentation toute différente : le fonctionnement par lectio, centrée sur telle ou telle quœestio, y est dominant. Il s'agit là d'un format oratoire organisé autour d'au moins trois moments discursifs (dico, objectio, respondeo), par la dynamique desquels le commentateur est en réalité contraint de choisir explicitement entre les autorités qu'il mobilise ${ }^{65}$. En mêlant le contenu de la qucestio de cours à la forme très écrite et très documentée du commentaire d'apparat, l'interprétation que Robortello donne des lignes 
1449b 24 à 28 de la Poétique subvertit simultanément les règles de ces deux types d'énonciation. Au lieu de produire une lecture qui puisse prétendre à être définitive, le commentaire imprimé en reste à une quœstio irrésolue. Ce faisant, l'impression des Explicationes fige à jamais en posture d'autorité un discours forgé pour demeurer instable par son contenu et inassignable dans sa discursivité.

\section{Écriture et actions} l'espèce d'« équivoque »? À vrai dire, tant que Robortello n'a pas quitté le patronage de Cosme, le dispositif énonciatif forgé par lui pourrait tout aussi bien être étiqueté "perplexité d'érudit ». Faut-il dès lors penser qu'en résolvant l'oscillation du texte par une action qui serait sans ambiguïté, seul le départ de Robortello pour Venise aurait, dans la rétrospection, fait apparaître l'équivoque - tout en la dissipant par là même ? Se posent ici deux questions. La première est celle des rapports entre une écriture et des actions. Cette question demeure insoluble tant qu'on n'envisage pas l'écriture comme une manière d'agir, à analyser parmi d'autres formes possibles d'action. La seconde question est celle des spécificités de l'écriture en tant que manière d'agir, qui conduit notamment à s'interroger sur l'écriture comme action persistante, parce que survivant aux contextes de sa production, comme aux modalités de sa circulation première.

Dans le discours para-universitaire qu'il prononça en 1549 pour remercier le sénat de l'avoir nommé à la Scuola di San Marco, Robortello évoque la «tyrannie » avec horreur, puis exhorte les sénateurs à tout faire pour maintenir la république, qu'il est selon lui de leur devoir de protéger des complots et d'affermir par l'éducation ${ }^{66}$. Pour se faire pardonner d'avoir officié six années dans le Studio Pisano - sous le patronage de ce que, à Venise, on appelle un "tyran »-il précisa aussi qu'il avait ignoré les surenchères financières de Cosme, qui aurait cherché à le retenir ${ }^{67}$. Il dit également son bonheur d'avoir retrouvé (lui qui était né à Udine) le patronage de la république et d'être chargé d'y faire fleurir la vertu par la diffusion des humanités :

Mais j'ai toujours été un adorateur de votre République, et je me souviens de moimême, dans le profondeurs de votre territoire, éduqué sous vos très saintes lois, et ne voulant manquer à aucune de vos exigences, surtout pour ce qui est de l'éduction de votre jeunesse à la vertu par le moyen des lettres. Ah, vraiment, quel bonheur est le mien ! O labeurs fortunés, si, une fois moi-même outillé [de savoirs], mon enseignement attisait en quelque manière ce qui alimente, préserve, et développe la patrie et si, suivi [dans mes conseils], mes admonestations étaient elles-mêmes couronnées de gloire..$^{68}$

Le choix de Robortello ne doit pas être compris au travers d'une dichotomie simpliste qui opposerait liberté et «tyrannie ». Car le sénat vénitien n'était pas moins scrupuleux que Cosme sur les formes que devait prendre le service politique des lettrés qu'il nommait : il l'était d'une manière différente, mettant notamment l'accent non pas sur un pouvoir personnel, comme celui de Cosme, mais bien sur la nécessité de promouvoir les multiples éléments du mythe politique de la sérénissime République, dont la virtù des patriciens était un élément central. Or Platon s'acclimatait sans doute mieux dans un tel cadre. Ou plutôt Venise permettait plus aisément à Robortello de se faire l'avocat d'une position syncrétique, inspirée de Platon comme d'Aristote, et centrée sur l'exaltation d'un régime politique où la vertu est posée comme essentielle. C'est ainsi qu'il faut comprendre la publication, en 1552, sous la direction de Robortello, d'un ensemble composite 
comportant une Francisci Philephi De Morali Disciplina libri quinque, une traduction du commentaire d'Averroès sur la République de Platon (Averrois Paraphrasis in libros De Republica Platonis) et un texte intitulé Francisci Robortelli In Libros Politicos Aristotelis Disputatio $^{69}$. Ce dernier texte (p. 169-178), intitulé dans le corps du texte Francisci Robortelli Utinensis De fine et materie politicoe scientio, seu artis disputatio Venetiis habita, MDL., cum libros Politicos Aristotelis publ. interpretaturus esset est en fait la transcription d'un discours adressé en 1550 aux sénateurs vénitiens, où Robortello exhorte les patriciens à la vertu politique, en se basant librement sur une lecture « républicaine » et anti-tyrannique de la Politique d'Aristote. L'érudit y distingue les «hommes politiques» qui agissent pour le bien de la cité et les «tyrans » qui agissent pour leur seul intérêt (p.172) et vante sans cesse l'importance de la morale en matière politique - élément que le texte de Filefo avait déjà abondamment souligné. Dans ce dernier discours, comme dans l'ensemble formé par cette succession de textes, ce sont les règles de l'oligarchie vertueuse qui sont énoncées.

Si l'on admet que Robortello préparait, en autres choses, son éventuel adoubement par le sénat vénitien en publiant ses Explicationes, on pourrait être tenté de croire que seules ses prises de positions tonitruantes contre la «tyrannie» seraient susceptibles de nous alerter sur la présence d'une équivoque dans son commentaire des lignes $1449 \mathrm{~b} 24$ à 28 de la Poétique. Mais cette lecture rétrospective a l'inconvénient majeur de faire disparaître l'équivoque en prétendant la démasquer: si Robortello n'a écrit que pour séduire les patriciens vénitiens, son écriture n'est plus équivoque, elle est dissimulée. L'écriture de Robortello est et demeure équivoque parce que, au moment où il fit paraître son commentaire, il cherchait tout autant à séduire Florence que Venise. Peut-être même hésitait-il encore quelque peu entre une vision platonicienne de la tragédie, et une conception plus aristotélicienne de ses usages? À moins que d'autres documents surgissent des archives italiennes, on ne saura probablement jamais si, alors qu'il rédigeait son commentaire, Robortello vomissait la «tyrannie » de Cosme dans le secret de son cabinet, ou bien s'il se résolvait avec pragmatisme à la nécessité de courtiser plusieurs patrons à la fois. Il y a donc plus. En l'espèce, les actions de Robortello demeurent aussi impénétrables que son écriture-parce que l'écriture n'est dans le présent cas qu'une forme d'action parmi d'autres possibles.

\section{«Catharsis »: De l'équivoque au Malentendu}

Mais c'est en quoi l'équivoque est aussi - mais non seulement - affaire de lecture ${ }^{70}$. Pour la percevoir, et aspirer éventuellement à la réduire, il n'est pas toujours nécessaire de la chercher, mais il faut néanmoins accepter d'être surpris (et parfois même séduit) par le dispositif rhétorique au sein duquel elle se déploie. Il faut, en somme, s'autoriser à se laisser piéger par l'énonciation d'une ambivalence.

[...] In vero i letterati per intenderla [la Poétique] perfettamente hanno dove ricorrere a'dotti scritti nuovamente mandati fuori di Messer Francesco Robortello, il quale di tal sorte fatto aperta quest'Opera, che nessuna oscurità più ci resti [...],

écrit Bernardo Segni dans sa paraphrase au premier chapitre du texte, parue, avec sa traduction, en $1550^{71}$. Il s'agit pour lui de renvoyer les plus lettrés de ses lecteurs au commentaire érudit de Robortello, en présentant son propre texte comme une simple glose. Mobilisant l'ethos philologique, la formule associe tout naturellement l'érudition des doctes avec la production d'un texte si «ouvert» ou clair, qu'il n'y resterait plus aucune obscurité. Si de nombreux passages des Explicationes peuvent correspondre à la 
description enthousiaste qu'en donne Segni, le commentaire de Robortello sur les lignes 1449b 24 à 28 de la Poétique pourrait néanmoins difficilement être tenu pour une exemplification de cet idéal. Alors que Robortello a, depuis un an, quitté Florence pour Venise, Segni continuait cependant à déchiffrer l'ensemble de son commentaire dans le contexte du patronage de Cosme, dont lui-même n'a pas encore perdu la faveur. Ecrivant pour ce public de notables que l'entourage du prince espérait convertir aux mérites de l'aristotélisme politique, Segni passe sous silence les obscurités du commentaire de la " catharsis ", ignorant (ou récusant ?) son hétérodoxie potentielle.

Nous possédons néanmoins au moins un témoignage de ce que le commentaire que Robortello donna des lignes des 1449b 24 à 28 de la Poétique fut compris par certains de ses lecteurs comme une construction ambivalente ${ }^{72}$. On le trouve dans la manière dont Pierre Corneille utilise et rend compte de sa lecture des Explicationes dans ses Trois discours sur le poëme dramatique (1660). Doutant de l'efficacité et même de la réalité de la " catharsis ", Corneille écrit dans son deuxième Discours :

J'avouerai plus. Si la purgation des passions se fait dans la tragédie, je tiens qu'elle doit se faire de la manière que je l'explique ; mais je doute si elle s'y fait jamais, et dans celles-là même [les tragédies] qui ont les conditions que demande Aristote. [...] Un des interprètes d'Aristote veut qu'il n'ait parlé de cette purgation des passions dans la tragédie, que parce qu'il écrivait après Platon, qui bannit les poètes tragiques de sa république, parce qu'ils les remuent trop fortement. Comme il écrivait pour le contredire, et montrer qu'il n'est pas à propos de les bannir des Etats bien policés, il a voulu trouver cette utilité dans ces agitations de l'âme, pour les rendre recommandables par la raison même, sur qui l'autre se fonde pour les bannir. Le fruit qui peut naître des impressions que fait la force de l'exemple lui manquait: la punition des méchantes actions, et la récompense des bonnes, n'étaient pas de l'usage dans son siècle, comme nous les avons rendues de celui du nôtre : et n'y pouvant trouver une utilité solide, hors celle des sentences et des discours didactiques, dont la tragédie peut se passer selon son avis, il a en substitué une qui n'est peut-être qu'imaginaire. Du moins si pour la produire il faut les conditions qu'il demande, elles se rencontrent si rarement que Robortel ne les trouve que dans le seul CEdipe, et soutient que ce philosophe ne nous les prescrit pas comme si nécessaires, que leur manquement rendent les ouvrages défectueux, mais seulement comme des idées de la perfection des tragédies. ${ }^{73}$

La référence à Robortello n'est explicite que dans la dernière phrase de cette réflexion, où il est fait allusion à un passage des Explicationes dont l'équivoque est absente ${ }^{74}$. Mais lorsque Corneille mentionne " un des interprètes d'Aristote ", l'ambivalence est totale. L'expression peut d'abord sembler renvoyer à la première phrase de Proclus, citée en grec par Robortello, dans laquelle le commentateur grec suggérait que l'affirmation platonicienne de la paradoxale nécessité de bannir les poètes avait poussé Aristote à inventer des arguments pour le contredire. Mais Proclus n'est pas un «interprète » d'Aristote, c'est un commentateur de Platon. Seul Robortello pourrait légitimement prétendre au titre d'«interprète» d'Aristote. L'expression utilisée par Corneille superpose donc la figure de Robortello et celle de Proclus, soit parce que le dramaturge n'ose mentionner le nom de Proclus dans un texte liminaire par lequel il espère toucher un public autre que les doctes et les érudits, soit - plus vraisemblablement - parce que, pour Corneille comme pour nous, le système énonciatif adopté par l'auteur des Explicationes ne permettait pas de conclure avec certitude ni à l'accord de Robortello avec Proclus, ni à leur désaccord. L'expression « un des interprètes d'Aristote » attribue donc implicitement la position de Proclus à Robortello lui-même, tout en respectant l'équivoque du texte des Explicationes. Plus de cent ans après sa rédaction, ce texte, sorti 
du contexte étroit que constituait le patronage de Cosme, est ainsi appréhendé par Corneille dans sa pleine et originelle équivocité.

Mais, alors même que Corneille, s'appuyant sur Robortello et sur Proclus, produit une contextualisation de l'énonciation d'Aristote (qui n'aurait inventé la «purgation des passions » que pour contredire Platon, parce qu'il ne pouvait mobiliser à cette fin, comme le fait Corneille lui-même, la notion de « force de l'exemple »), l'auteur des Trois discours sur le poëme dramatique ne suggère aucune lecture contextualisée du discours de Robortello (ou de Proclus). Détaché du maillage d'actions dans lequel il fut produit, le texte de Robortello se trouve revêtu de l'autorité, tout autant philologique que philosophique, que lui donne, du point de vue de Corneille, son statut de premier d'une longue série de commentaires savants de la Poétique d'Aristote. C'est dire qu'alors qu'il est vraisemblable que l'équivocité du commentaire de Robortello sur la notion de « catharsis » soit largement imputable à sa recherche simultanée de plusieurs patronages, la lecture qu'en donne Corneille reporte l'équivocité du texte de Robortello sur celui d'Aristote. Si Robortello (mais aussi Proclus) ont vu dans ce passage de la Poétique matière à quosstio, ce ne peut être, apparemment, que parce que ce texte serait intrinsèquement problématique. Tandis que, aux yeux d'Averroès, la "catharsis» était peut-être une notion si limpide qu'elle n'appelait nulle glose, le terme devient, sous la plume de Corneille, si obscur, que le dramaturge propose purement et simplement de se débarrasser d'une notion qu'il construit en obstacle à la bonne intelligence des utilités de la tragédie. C'est que, comme Robortello avant lui - mais aussi comme Aristote, Proclus, et Averroès avant eux - Corneille poursuit ses propres buts en s'appropriant la notion de « catharsis », qu'il s'empresse de remplacer dans ses Discours par celle d'« exemple d $^{75}$. En l'occurrence, il s'agit pour lui de révoquer en doute une notion dont les poéticiens à la solde de Richelieu avaient fait la cheville ouvrière de leur conceptualisation de l'utilité politique du théâtre, et à laquelle lui-même ambitionne de substituer une palette d'usages plus moraux que civils ${ }^{76}$.

On pourrait poursuivre l'histoire des réappropriations de la notion de « catharsis » jusque dans les dernières parutions prétendant aujourd'hui éclaircir, tout autant qu'utiliser, le texte $\mathrm{d}^{\prime}$ Aristote $^{77}$. La tâche serait infinie ${ }^{78}$. On se bornera donc à constater qu'une écriture elliptique (celle du texte d'Aristote dans l'état où il nous est parvenu) et une série d'écritures critiques et/ou équivoques semblent avoir, en l'espèce, l'étrange pouvoir de générer un malentendu après l'autre. La lecture esthétisante de la "catharsis ", qui domine l'interprétation de la Poétique d'Aristote sur la scène intellectuelle française depuis la parution, en 1980, de la traduction annotée de la Poétique par Roselyne DupontRoc et Jean Lallot, n'est que l'un des derniers avatars de cette longue série ${ }^{79}$. Dans cette dernière lecture, qui inscrit Aristote dans le «régime esthétique des $\operatorname{arts}^{80} »$, le " sentiment esthétique " est présenté comme la solution à un "problème " philologique et philosophique qui n'en est un que depuis Robortello. Qui voudra être généreux dira depuis Proclus. Ce qui, à vrai dire, ne changerait pas grand chose à l'affaire puisque, sans Robortello, Proclus resterait aujourd'hui à peu près totalement oublié de tous ceux que la « catharsis » fascine.

Du point de vue de l'histoire sociale et politique des discours sur la poésie et les arts, l'essentiel reste l'extraordinaire productivité théorique de ce qui est avant tout une série de pataquès greffés sur un texte équivoque. On en citera un dernier exemple, par simple plaisir pris à la circularité. Dans leur préface à une récente édition des Trois discours sur le 
poème dramatique de Corneille, Bénédicte Louvat et Marc Escola soutiennent que, dans les réflexions de Corneille sur la « catharsis",

[...] il s'agit en fait [pour lui] d'orchestrer la disparition de la catharsis, telle du moins qu'elle a été comprise par les commentateurs d'Aristote aux $\mathrm{XVI}^{\mathrm{e}}$ et $\mathrm{XVII}^{\mathrm{e}}$ siècles, du champ de la poétique. [Selon eux], la catharsis n'est convoquée [par Corneille] au titre de quatrième "utilité » que pour mieux mettre en question l'interprétation moralisante donnée au $\mathrm{xVI}^{\mathrm{e}}$ siècle au concept aristotélicien, compris alors en référence aux préceptes de l'utile dulci formulés par Horace: Corneille se montre plus «aristotélicien » que bien des interprètes d'Aristote en cherchant à énoncer la nature spécifique de l'émotion tragique, une émotion non pas immédiatement morale, mais pleinement esthétique et qui n'a pas d'autre finalité qu'elle-même ${ }^{81}$.

Aristote et Corneille seraient donc des kantiens de la plus stricte obédience. Le croira qui voudra.

\section{NOTES}

1. Francisci Robortelli Vtinensis in librum Aristotelis De Arte poetica explicationes. Qui ab eodem authore ex manuscriptis libris, multis in locis emendatus fuit, ut iam difficillimus, ac obscurissimus liber a nullo ante declaratus facile ab omnibus possit intelligi, suivi de Francisci Robortelli Utinensis Paraphrasis in librum Horatii qui vulgo De Arte poetica ad Pisones inscribitur. Ejusdem explicationnes de satyra, de epigrammate, de commoedia, de salibus, de elegia, quœ omnia addita ab authore fuerunt, ut nihil quod ad poeticam spectaret desiderari posset. Nam in iis scribendis Aristotelis methodum servavit, \& ex ipsius libello De Arte poetica principia sumsit omnium suarum explicationum, Florentiæ, In Officina Laurentii Torrentini Ducalis Typographi, 1548, cum Summi Pontif. Caroli V Imp. Henrici II. Gallorum Regis, Cosmi Medecis Ducis Florent. II. Privilegio, in-folio. Une seconde édition parut à Bâle en 1555, chez Giovanni Ervagio le Jeune. Pour une réimpression de l'édition de 1548, voir W. Fink Verlag, Poetiken des Cinquecento, Bd 8, Münich, 1968. La phrase citée se trouve à l'ouverture de l'Ad lectorem. Ce livre sera ci-après désigné sous le titre d'Explicationes.

2. Voir Antony Grafton, Joseph Scaliger : A Study in the History of Classical Scholarship, 2 vol., Oxford, Clarendon Press, 1983, t. 1, p. 9-70.

3. Pour l'unique notice biographique concernant Robortello, voir Gian Giuseppe Liruti, Notizie delle Vite ed Opere scritte da Letterati del Friuli [...], Venise, Modesto Fenzo, 1762, 4 volumes, réédition à l'identique à Bologne, par Forni Editore, 1971. La vie de Francesco Robortello se trouve aux pages 413-483 du tome II. Sur le statut des professeurs et la compétition académique, voir Paul F. Grendler, The Universities of the Italian Renaissance, Baltimore \& London, Johns Hopkins University Press, 2002, p. 159-166. Pour une analyse des travaux philologiques de Robortello, voir Antonio Carlini, "L'attività filologica di Francesco Robortello », Udine, Accademia di Scienze Lettere e Arti di Udine, texte extrait des Atti dell'Academia di Udine, 1966-1969, serie VII, vol. VII, Arte Grafiche Friulane, 1967, p.6-36. Les rivalités éditoriales se prolongeaient parfois en polémiques savantes. Le nom de Robortello reste ainsi attaché à une violente dispute académicophilologique qui l'opposa à Carlo Sigonio, tenant en haleine l'ensemble de l'université de Padoue pendant toute l'année 1562. Voir sur ce point William MacCuaig, Carlo Sigonio : The Changing World of the Late Renaissance, Princeton, Princeton University Press, 1989, p 25-28 et p. 43-50. 
4. Sur le statut de ces chaires de studia humanitatis, le contenu de cet enseignement et son évolution, voir Paul F. Grendler, op. cit., p. 199-248.

5. Sur le statut de la philosophie morale dans les universités du Cinquecento, voir notamment Antonio Poppi, «Il problema della filosophia morale nelle scuola padovana del Rinascimento: platonismo et aristotelismo nella definizione del metodo dell'etica » dans Platon et Aristote à la Renaissance, $\mathrm{XVI}^{\mathrm{e}}$ colloque international de Tours, Centres d'Études Supérieures de la Renaissance, Paris, Vrin, 1976, p. 105-146 ainsi que D. A. Lines, Aristotle's Ethics in the Italian Renaissance (ca. 1300-1650) : the Universities and the Problem of Moral Education, Leiden, Brill, 2002.

6. Le parcours de Pier Vettori, à en juger seulement par les jalons que constituent ses publications, diffère ainsi fort peu de celui de Robortello. Voir sur ce point Petri Victorii Commentarit in tres libros de Arte dicendi [...], Florentiæ, in officina B. Junctæ, 1548; Petri Victorii Commentarit in primum librum Aristotelis de Arte poetarum [...], Florentiæ, ex officina Juntarum, Bernardii filiorum, 1560 ; Petri Victorii Commentarii in libros Aristotelis de Optimo statu civitatis [...], Florentiæ, apud Juntas, 1576; Petri Victorii Commentarii in libros X Aristotelis de Moribus ad Nichomachum [...], Florentiæ, ex officina Junctarum, 1584. On peut penser aussi aux parcours de Jason de Nores ou de Faustino Summo. Je me permets de renvoyer, pour l'étude ces deux dernières carrières, à mon propre article, «Jean Chapelain et les 'lumières de Padoue' : l'héritage italien dans les querelles françaises sur l'utilité du théâtre (1585-1640) », Littératures classiques, $\mathrm{n}^{\circ}$ 37, 1997, p. 97-116.

7. L'ensemble des indications biographiques données ici sont tirées de Gian Giuseppe Liruti, Notizie [...], op. cit.. Soigneuse, jusque dans son usage des correspondances et des archives, cette étude biographique date néanmoins de 1762 : elle mériterait d'être affinée et complétée.

8. Notizie [...], op. cit., p. 419. Certaines de ces oraisons ont survécu sous forme manuscrite.

9. Ibid., p. 420.

10. Sur ces événements et les premières années du pouvoir de Cosme $\mathrm{I}^{\mathrm{er}}$, voir Eric Cochrane, Florence in the Forgotten Centuries 1527-1800: A History of Florence and the Florentines in the Age of the Grand Dukes, Chicago et Londres, Chicago University Press, 1973, p. 13-66.

11. British Library Add. Mss. 10.265 et 10.276 .

12. Explicationes, op. cit., dédicace non paginée : «Ac sane FRANC. CAMPANUS, vir propter singularem sapientiam, et virtutem, atque admirabilem doctrinam omni memoria dignus, [...], quod is mihi in clientelam tuam primus aditum patefecit [...]». Dès 1538, Campana avait confié à Pier Vettori une chaire d'humanités au Studio Fiorentino, avec un très confortable salaire annuel de 300 florins (voir Dizionario biographico degli Italiani, tome 17, Rome, Socièta Graphica Romana, 1974, p. 344).

13. Francisci Robortelli Utinensis variorum locorum Annotationes tam in Grœeis, quam Latinis authoribus, cum Gratia \& Privilegio Senatus Veneti, Venetiis apud lo. Baptistam Burgofrancho Papiensem, 1543; Francisci Robortelli [...] Variorum locorum annotationnes tam in grœcis, quam latinis authoribus, Parisiis, apud N. Boucher, 1544 et Francisci Robortelli Utinensis In varia loca quœ tam in Grœcis scriptoribus quam in Latinis passim leguntur Annotationum libri duo, Florence, Lorenzo Torrentino, 1548.

14. Notizie [...], op. cit., p. 421.

15. Je remercie Caroline Callard de m'avoir signalé ce fait. Voir sur ce point le premier chapitre de sa thèse, Storia Patria : histoire, pouvoir et société à Florence au XVII siècle, soutenue à l'université de Paris-IV en 2001, A.N.R.T., Lille, 2003.

16. Sur Torrentino, voir Antonio Ricci : «Lorenzo Torrentino and the Cultural Programme of Cosimo I de' Medici », dans Konrad Eisenbichler (éd), The Cultural Politics of Duke Cosimo de' Medici, Aldershot, Ashgate, 2001, p. 102-119.

17. Bernardo Segni, Tratiato dei Governi di Aristotile, tradotto di greco in lingua vulgare fiorentina da Bernardi Segni, gentil'huomo e Accademico fiorentino, Firenze, Torrentino, 1549, Rettorica et Poetica d'Aristotele tradotta di greco in lingua vulgare fiorentina da Bernardi Segni, Florence, Torrentino, 1549 (réédition en 1551), L'Ethica d'Aristotile tradotta in lingua vulgare fiorentina et comentata per Bernardo Segni, Florence, Torrentino, 1550. 
18. Trattato dei Governi, op. cit., p. 6-8.

19. L'Ethica, op. cit., p. 3-5 et suivantes.

20. Voir Rettorica et Poetica, op. cit., préface à la traduction de la Poétique, p. 165.

21. Sont mentionnés : le privilège du Souverain Pontife, celui de Charles Quint, celui d'Henri II de France et enfin celui de Cosme.

22. Bernard Weinberg, A History [...], tome 1, op. cit., p. 383 l'affirme, citant sans autre précision, en note 50, un manuscrit de la Biblioteca Nazionale di Firenze (Ms BNF II.IV.192) que je n'ai pas encore pu consulter. S'il fut effectivement présenté devant l'académie florentine, le texte de cette leçon ou introduction devait être en italien.

23. Explicationes, op. cit., p. 52-55.

24. Voir De Arte poetica : translatio Guillelmi de Moerbeka suivi de l'Expositio media Averrois sive Poetria Hermanno Alemanno Interprete, édités par Lorenzo Minio-Paluello, dans Aristoteles Latinus; XXXIII bis (Corpus philosophorum Medii Ævi), Bruges, Desclée de Brouwer, 1968.

25. À titre indice, les collections de la Bibliothèque nationale de France répertorient huit manuscrits grecs de la Poétique, dont un seul (Fonds Grec 1741) date des $\mathrm{x}^{\mathrm{e}}-\mathrm{XI}^{\mathrm{e}}$ siècles. Cinq des huit manuscrits présents dans cette collection sont des textes $d u \mathrm{xV}^{\mathrm{e}}$ siècle (Fonds Grec 2038, 2040, 2551, 2958, et le manuscrit $324 \mathrm{du}$ fonds Coislin) et furent probablement copiés en Italie ou en France sur des manuscrits ramenés de Byzance vers 1450-1470, ou sur des copies, italiennes ou françaises, de ces manuscrits byzantins. On trouve seulement un manuscrit du $\mathrm{XvI}^{\mathrm{e}}$ siècle (Fonds Grec 2117) et un du XVII ${ }^{\mathrm{e}}$ siècle (Supplément Grec 488). Dans la plupart des cas, la Poétique d'Aristote a été copiée et/ou reliée avec des manuscrits de la traduction rhétorique et poétique grecque : Dionysil Halicarnassei ars rhetorica, Dionysit Halicarnassei liber de verborum compositione, Menandri rhetoris de genere demonstrativo libri II, Demetrii Phalerei opusculum de elocutione mais aussi des oraisons de Démosthène ou même la Théogonie d'Hésiode. Pour plus de détails, voir Henri Omont, Inventaire sommaire des manuscrits grecs de la Bibliothèque nationale, 4 volumes dont le premier contient l'index, Paris, Alphonse Picard, 1886-1898, réédition Georg Olms Verlag, Hildesheim/Zurich/New York, 2000.

26. Explicationes, op. cit., Ad lectorem : "Quatuor enim ego usus sum libris ; tribus manuscriptis, quorum duo sunt in Medico Bibliotheca; aller quidem Politiani manu descriptus; alter vero multo vetustior; plurimum autem tribuendum \& illi puto, propter Politiani singularem doctrinam, \& acre judicium, viri mehercle cum antiquis conferendi. »

27. Cette traduction est parue dans un volume de miscellanées, Logica cum aliis aliorum operibus, Georgio Valla interprete, Venitiis, per Simonem Papiensem dictum Bevilaquam impressum, 1498. Le livre comprend: la Logique de Nicéphore, un texte de G. Valla sur l'argumentation, les Eléments d'Euclide, un texte de Proclus sur l'astrolabe, un De Mundo attribué à Timeus, un autre attribué Cleomedes, le De Coelo, la Magna Ethica et l'Ars Poetica d'Aristote, puis des textes de médecine attribués à Rhazès, Galien et Alexandre d'Aphrodise. Giorgio Valla se voit attribuer des « explications » (G. V. explicit) sur le De Colo, la Magna Ethica, et l'Ars poetica, ainsi que sur certains textes de Galien, d'Alexandre d'Aphrodise et de Rhazès. Valla enseignait le grec, les sciences et la rhétorique à la Scuola di San Marco et était très lié avec Manuce (voir M. Lowry, The World of Aldus Manutius: Business and Scholarship in Renaissance Venice, Oxford, Basil Blackwell, 1979, p. 181-216, 183-184 et 191-194). Valla fournissait à Manuce des manuscrits et faisait fréquemment de la publicité pour les éditions aldines dans ses cours. On voit difficilement comment Manuce n'aurait pas pu obtenir de Valla une copie d'un manuscrit de la Poétique, que Valla possédait. Bref, l'hypothèse qu'Aide n'ait pas publié ce texte (et la Rhétorique) parce qu'il n'aurait pu obtenir des copies de ces manuscrits est peu convaincante. C'était celle d'A. Firmin-Didot, dans Alde Manuce et l'hellénisme à Venise, Paris, Typographie d'Ambroise Firmin-Didot, 1875, p. 103-105. Il s'appuyait sur la dédicace de Manuce à Alberto Pio dans le dernier tome de son édition d'Aristote, où Manuce se plaignait de n'avoir pu réunir tous les manuscrits aristotéliciens recherchés. Mais peut-être Manuce n'avait-il en réalité guère d'intérêt (philosophique tout autant que 
commercial) pour ces deux textes : son édition était centrée sur la science et la philosophie, la poésie et la rhétorique n'étant considérées que comme des savoirs propédeutiques. A. Bondini, dans le colophon du premier volume de l'édition aldine (1495) explique ainsi qu'après avoir longtemps vénéré la poésie et la rhétorique, lui-même a pris goût à des savoirs solides - soit plus proches de l'Être - et invite le lecteur, s'il veut être heureux, à faire de même en lisant Aristote dans les volumes aldins (ibid., p. 67-68). La Poétique et la Rhétorique d'Aristote parurent pour la première fois en grec dans le premier des 2 vol. intitulés Rhetores grœci [...], Venise, Aldus Manutius, 1508, soit dans une série consacrée exclusivement à la tradition rhétorique grecque, qui se trouve ainsi comme isolée de la philosophie et des sciences à proprement parler. Ces deux volumes comprennent en particulier les textes suivants: Hermogenis ars Rhetorica, Dionysii Halicarnassei ars Rhetorica, Demetrii Phalerei de interpretatione, Menandri Rhetoris divisio causarum in genere demonstrativo. À en juger par ce dont la Poétique se trouve ici entourée, la première publication imprimée de ce texte s'inspire donc très largement de la tradition qui se donnait à voir dans les manuscrits grecs alors disponibles, soit d'un ensemble d'usages où le texte d'Aristote est perçu comment un élément du canon rhétorique et poétique transmis par les Grecs (voir note 26). On remarque que, dans cette tradition manuscrite, l'association de la Poétique avec les textes des grands tragiques grecs, qui nous semble aujourd'hui évidente en raison du contenu même du texte, brilla en réalité par son absence.

28. Aristotelis Poetica, per Alexandrum Paccium, Patritium Florentium, in latinum conversa, Venetiis, in Aedibus haeredum Aldi, et Andrece Asulani Soceri, 1536. Voir aussi Aristotelis De Rhetorica libri tres, De eadem ad Alexandrum liber unus, De Poetica liber unus, Venetiis in cedibus Batholomæi Zanetti, cere et diligentia Ioannis Francisci Trincavelli, 1536, où la traduction latine est également celle de Pazzi.

29. Voir Richard S. Samuels, «Benedetto Varchi, the Accademia degli Infiammati, on the Origins of the Italian Academic Movement ", dans Renaissance Quarterly, n²9, 1976, p. 599-633, p. 614 en particulier et Bernard Weinberg, A History of Literary Criticism in the Italian Renaissance, Chicago, Chicago University Press 1961, 2 vol., tome 1, p. 373. Lombardi étant mort peu de temps après, Maggi décida de continuer ses travaux sur la Poétique, qu'il fit paraitre sous leurs deux noms en 1550 avec le titre de Vincentii Madii Brixiani et Batholomeo Lombardi Veronensis in Aristotelis Librum De Poetica communes explanationes [...], Venitiis, in officina Erasmiana Vincentii Valgrisi, 1550, réimpression par W. Fink Verlag, Poetiken des Cinquecento, Bd 4, Munich, 1969.

30. Citée par Bernard Weinberg, Ibid.

31. La rivalité entre les deux éclata en 1550 lorsque Maggi, sans doute furieux d'avoir été devancé par Robortello, publia ses De Poetica communes explanationes en y faisant figurer des Objectiones quoedam adversus Robortelli explicationem in primum Aristotelis contextum, op. cit., p. 16-26.

32. Ce dont, écrit Robortello, Francesco Campana pourrait témoigner, s'il vivait encore (il est mort en 1546) ; Explicationes, op. cit., dédicace non paginée.

33. Ibid : "[...] fuerat ab Aristotele, qui philosophice omne genus erat tractatus, multo ante descripta, sed rhetoricen, \& poeticen, quœe duœ artes \& maximœ, \& prœclarissimœ; Dii boni, quam apte, quam distincte,

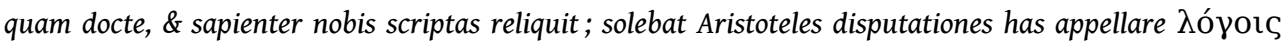

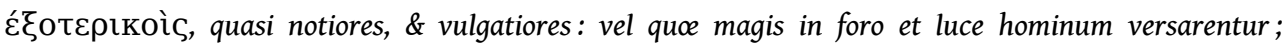
huiusmodi Aristotelis libros, qui de Rhetorice et Poetice circunferuntur, non video ab iis, qui nostra hac cetate Aristotelei philosophi vocantur, multum legi, aut tractari, neque vero propter obscuritatem crediderim ; multo enim obscuriora illustrant, sed aut quod leviora putant, quam ut in iis operam suam ponere velint, aut quod nonnullarum rerum cognitione, \& ea quidem permediocri, contenti, ea negligunt, quoe ad ornatum cultiorem, et pompam veluti quandam theatralem, \& forensem pertinent [...]». Je traduis.

34. Ibid. Tout ignorer de l'Antiquité, de son histoire comme de ses auteurs, était l'un des principaux reproches que les humanistes faisaient à l'approche scolastique.

35. À titre d'indice de ce statut ancillaire, soulignons que dans toutes les éditions complètes du corpus aristotélicien publiées en Europe entre 1530 et 1670, la Poétique figure après les deux textes 
rhétoriques attribués à Aristote, soit après la Rhétorique à Théodecte et la Rhétorique à Alexandre, même si la place attribué à ce groupe de textes dans l'ensemble de l'œuvre d'Aristote peut varier considérablement selon les éditions. Robortello, dans l'épître dédicatoire de son traité, explique qu'il était naturel, dans le cours de son enseignement à Pise, de passer de la rhétorique à la poétique.

36. Sur la tradition de l'inventio rhétorique, voir Mary J. Carruthers, The Craft of Thought: méditation, Rhetoric and the Making of Images (400-1200), Cambridge, Cambridge U. P., 1998, trad. française par Françoise Durand-Bogært sous le titre de Machina memorialis : méditation, rhétorique et fabrication des images au Moyen Âge, Paris, Gallimard, 2002.

37. Voir sur ce point, Stephen Halliwell, Aristotle's Poetics, Londres, Duckworth, 1986 et 2000 pour la $3^{\mathrm{e}}$ éd. augmentée, ch. VI : « Tragedy and the Emotions », p. 167-201.

38. Voir Averroes' Middle Commentary on Aristotle's Poetics, traduit, annoté et présenté par Charles E. Butterworth, Princeton, Princeton University Press, 1986, ainsi que Deborah L. Black, Logic and Aristotle's Rhetoric and Poetics in Medieval Arabic Philosophy, Leiden, Brill, 1990 et Salim Kemal, The Poetics of Alfarabi and Avicenna, Leiden, Brill, 1991.

39. Les notes prises par un étudiant pouvaient par exemple circuler, et Bernard Weinberg, $A$ History [...], op. cit., p. 373-383, mentionne précisément un manuscrit de ce type : Alessandro Sardi prit des notes sur le cours que Vincenzo Maggi consacra à la Poétique en 1546 à l'université de Ferrare et ces notes subsistent dans la Biblioteca Estense à Modène sous la cote MS.a. Q.6.14.

40. De Poetica communes explanationes [...], op. cit., p. 97.

41. Ibid., p. 97-98.

42. Deux indices textuels pourraient signaler une imprégnation réciproque. Tout d'abord l'exemple de la puce (pulex), qui sert dans les deux textes à démontrer que la tragédie peut trouver en elle-même perfection et grandeur, puisque même un corps des plus petits, tel que celui de cet insecte, peut être parfait. Par ailleurs, Maggi et Robortello citent tous deux aussi le texte de la Politique VIII, 7. La succession des publications orales et imprimées a néanmoins pour conséquence de rendre insoluble la question de savoir qui s'est inspiré de qui.

43. Sur la prégnance de ce modèle dans les codes énonciatifs de l'époque moderne, voir Louis Marin, Le Récit est un piège, Paris, Minuit, 1978.

44. Explicationes, op. cit., p.53: "Quod si quis roget, qualis sit Aristotelis sententia de tragcedia. Respondeo, existimare illum; eius recitatione, \& inspectione purgari perturbationes has duos, commiserationem, \& metum. Dum enim homines intersunt recitationibus; audiuntque \& cernunt personas loquentes \& agentes ea, quœ multum accedunt ad veritatem ipsam, assuescunt dolere, timere, commiserari; quo sit ut cum aliquid ipsis humanitus acciderit, minus doleant, \& timeant, necesse est enim prorsus, ut qui nunquam indoluerit ob aliquam calamitatem, vehementius postea doleat, siquid adversi prœter spem acciderit. Adde quod sepe homines perperam dolent, ac timent; dum autem poetœ in recitationibus suarum tragcediarum offerunt personas, ac res dignissimas commiseratione, quasque iure unusquisque, vel sapiens, extimescat; discunt homines qualia sint ea, quœ iure commiserationem cieant, \& luctum, quœque metum incutiant. Postremo auditores, \& spectatores tragœdiarum hanc capiunt utilitatem, quœe prorsus maxima est, cum enim communis sit omnium mortalium fortuna, nullusque sit, qui calamitatibus non sit subiectus; facilius ferunt hominis, si quid adversi acciderit, eoque se solatio plane firmissimo sustenant, quod aliis etiam idem accidisse meminerint. " Je traduis.

45. Athénée, The Deipnosophists, VI, 223b-d, avec une traduction anglaise par Charles B. Gulick, 7 vol., Londres, William Heineman Ltd et New York, G. P. Putnam's Sons, 1929, vol. 3, p. 4-7.

46. Sur Athénée, qui restait mal connu, voir une somme récente : David Braund et John Wilkins (éd.), Athenaeus and his World: Reading Greek Culture in the Roman Empire, préface de Glen W. Bowersock, Exeter, University of Exeter Press, 2000.

47. Le texte mobilisé par Robortello est commodément accessible en français dans Proclus, Commentaire sur la République, notes et traduction par André Jean Festugière, 2 vol., tome I, 
dissertations I-VI, Paris, Vrin, 1970, V dissertation contenant une discussion des opinions de Platon sur la poésie, p. 60-85.

48. Explicationes, op. cit., p. 54.

49. Commentaire sur la République, op. cit., p. 67, 49.13 à 49.20, j'indique par des italiques les éléments du texte que Robortello cite en grec: "Le second problème - c'était ce bannissement paradoxal de la tragédie et de la comédie, paradoxal s'il est vrai qu'il est possible, grâce à elles de satisfaire en due proportion les passions et les ayant satisfaites, de les rendre capables de servir à l'éducation, parce qu'on a remédié à leur nocivité-, ce deuxième problème donc, qui a fourni a Aristote une ample occasion d'attaque, et aux défenseurs de ces genres poétiques prétexte à leurs écrits contre Platon, nous le résoudrons, quant à nous, à peu près ainsi en accord avec la doctrine précédente. "

50. Voir le texte de Robortello, Explicationes, op. cit., p. 54. Le Commentaire de la République, op. cit., p. 67, 49.20-50.5, donne: «Tout genre poétique qui imite les mœurs de toute sorte, qui, d'une part, à cause de l'imitation, pénètre aisément dans les esprits des auditeurs, d'autre part, à cause de la bigarrure, leur devient funeste - car quels qu'aient été les caractères imités, il est fatal que s'en revête celui qui se passionne pour ces imitations -, est absolument défavorable à la conduite des jeunes à la vertu. Car la vertu est chose simple et ce qui ressemble le plus à Dieu même, dont nous disons que l'attribut principal est l'unité. Celui-là donc, qui est destiné à ressembler à un tel être doit fuir la vie contraire à la simplicité, en sorte qu'il lui faudra rester pur de toute bigarrure. Il est donc clair que nous devrons nous garder de la tragédie et de la comédie qui imitent les mœurs de toutes sortes [...]. »

51. Commentaire de la République, op. cit., p. 67-68.

52. Je dois cette précision à Marwan Rashed. Cette lecture me semble infiniment plus convaincante que celle proposée par A. J. Festugière, Commentaire de la République, op. cit., p. 68, note 1 .

53. Explicationes, op. cit., p. 54.: «Duce autem causœ (ut summatim dicamus) impulerunt Platonem ut

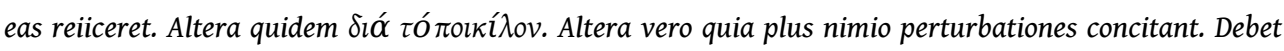

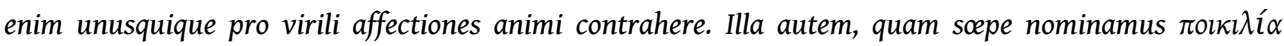
intelligitur, cum imitamur tam bonos, tam malos; \& continentes, \& incontinentes simul. Nam Plato non

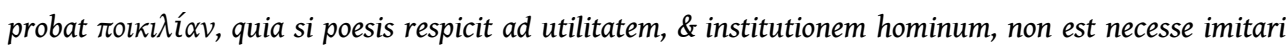
ullum, prœter bonos, \& sapientes viros. Nam (ut Proclus ait in quœstione prima) homme suapte natura

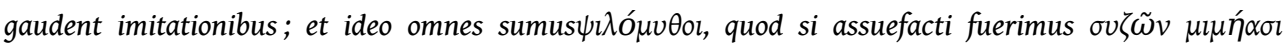

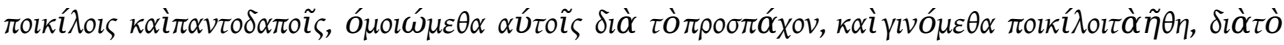

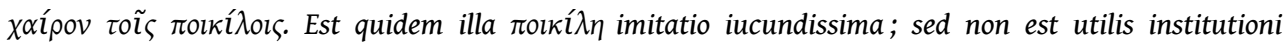
hominum. " Je traduis. La phrase citée en grec par Robortello correspond à un passage du Commentaire de Proclus qui est antérieur à ceux précédemment cités, op. cit., p. 64, 46.14 à 20 .

54. Avant 1550, le commentaire de la République par Proclus était accessible dans au moins une édition complète de Platon : Platonis Omnia opera, cum commentants Procli in Timcum et Politica [...], Basileæ, apud J. Valderum, 1534, 2 parties en 1 vol. in-folio, où la seconde partie contient les commentaires de Proclus.

55. Commentaire sur la République, op. cit., p. 66, 48.11 à 24 .

56. Pour une étude des portraits commandités par Cosme, voir Kurt W. Forster, « Metaphores of Rule: Political Ideology and History in the Portraits of Cosimo I de'Medici », dans Mitteilungen des

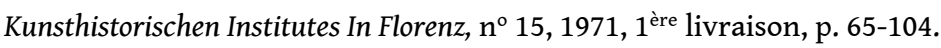

57. Sur Proclus et cette conception du mythe comme vérité énoncée à partir d'éléments fallacieux, voir Alain Lernould, Physique et théologie: lecture du Timée de Platon par Proclus, Villeneuve d'Ascq, Presses Universitaire du Septentrion, 2001.

58. Explicationes, op. cit., p. 55.

59. Cette volonté de créer une somme du savoir poétique appuyé sur deux autorités est lisible dès le titre du second élément de l'ensemble constitué par les Explicationes (consacré à une paraphrase d'Horace, suivie de traités sur divers genres non documentés dans les textes des 
Anciens ayant survécu), où Robortello vante son ouvrage comme un livre composé de telle sorte que « nihil quod ad poeticam spectaret desiderari posset ».

60. À titre d'indice de l'autorité et de la diffusion du Quomodo adulescens poetas audire debere (ou encore De audiendis poetis) signalons que le manuscrit de la Poétique d'Aristote annoté par Ange Politien - dont Robortello avait pu disposer pour préparer son édition - était relié avec deux autres textes alors tenus pour canoniques sur les questions poétiques, soit ce dialogue de Plutarque et De Elocutione de Démétrios de Phalère (voir Danilo Aguzzi-Barbagli, « Humanism and Poetics ", dans Renaissance Humanism : Foundations, Forms and Legacy, vol. 3, édité par Albert Rabil Jr., Philadelphia, Unversity of Pennsylvania Press, 1988, p. 97). D'une manière générale, l'établissement progressif d'un discours d'autorité sur la poésie et les arts semble s'être accompagné d'une modification profonde, voire d'un appauvrissement considérable, du système des références mobilisables sur un tel sujet: après 1600, Aristote et Horace - et de manière d'abord plus subversive et secrète, le traité du «sublime» du pseudo-Longin - écrasent toute autre autorité.

61. Pour une discussion du caractère platonicien de ce dialogue, voir Plutarque, Euvres morales, tome I, première partie, Paris, Les Belles-Lettres, 1987, notice du Comment lire les poètes, texte établi et annoté par André Philippon, p. 73-80. Pour une description plus générale des positions de Plutarque, voir Robert Lamberton, Plutarch, préface de John Herington, New Haven et Londres, Yale University Press, 2001.

62. C'est par exemple ainsi que procède l'oratorien Jean-François Senault dans son De l'Usage des passions (1641), où la dédicace flatteuse adressée à Richelieu ouvre sur un texte dans lequel le lecteur est libre de lire une condamnation implicite des ambitions du ministre. Je me permets de renvoyer à mon article, «Éloquence, mémoire et histoire : représentations de la guerre civile dans les écrits du R. P. Jean-François Senault" dans La Représentation de l'histoire au XVII siècle (mai 1997), textes réunis par Gérard Ferreyrolles, Publications de l'Université de Dijon, numéro CI, Dijon, Éditions Universitaires de Dijon, 1999, p. 77-103.

63. C'est ainsi que dans le commentaire qu'il donne de la nécessité de l'unité d'action (Poétique 1451a 16 à 35) et des polémiques qu'elle suscite, Robortello cite une contribution de Campana à ce débat et fait son éloge ainsi que celui des Médicis (voir Explicationes, op. cit., p. 85-86).

64. Sur les « arts de faire » et leur subversion, voir Michel de Certeau, L'Invention du quotidien : I. Arts de faire, nouvelle édition, établie et présentée par Luce Giard, Paris, Gallimard, « coll. Folio », 1990, notamment p. XXXIII à LIII.

65. Voir par exemple les fragments de notes d'un cours sur la Poétique d'Aristote donné par Carlo Sigonio quelque part entre 1552 et 1564 et publiés par William MacCuaig dans "Carlo Sigonio's lectures on Aristotle's Poetics ", Quaderni per la storia dell'Université di Padova, n 16, 1983, p. 43-69. Les notes de Sigonio ne sont pas un commentaire par particula, mais un commentaire isolant dans le texte des notions (ou quœstiones poétiques) telles que l'imitation, la prosodie, le rythme ou la rime : le texte d'Aristote ne sert que point de départ épisodique et Sigonio affirme parfois ses opinions par des verbes tels que declaro ou credo.

66. Francisci Robortelli Utiniensis Oratio Venetiis habita, antequam initium faceret interpretandi, pridie calen. Novem. M. D.XLIX, Venetiis, apud Petrum de Nicolinis de Sabio, 1549. Le texte est sans pagination.

67. Ibid., avant dernière page : «[...] certissimos, uberissimosque proventus, quos pro sua benignitate Maximus Reipublicoe Florentina Dux Cosmus, non solum ad sperandum, sed etiam ad fruendum proposuerat, neglexi. »

68. Ibid., dernière page : «Sed cum ego reipublicœ vestro amantissimus semper fuerim, ac meminissem me in vestris regionibus altum, sub vestris sanctissimis legibus educatum vobis petentibus deesse nolui, in prosertim tantam, ut vestram ivventutem literis instituere ad virtutem. 0 me vero felicem! 0 fortunatos labores meos, siquis aliquando extiterit mea disciplina instructus, qui patriam adjuvet, conservet, augeat, \& meas admonitiones secutus gloriam consequantur. » 
69. Francisci Philephi De Morali Disciplina libri quinque; Averrois Paraphrasis in libros De Republica Platonis ; Francisci Robortelli In Libros Politicos Aristotelis Disputatio, cum Privilegio Illustriss. Senatus Veneti ad Decennium, Venetiis, 1552 (nouvelle édition à l'identique en 1578 : seule la page de titre diffère).

70. Sur la prégnance de "malentendus" dans les discours des sciences humaines et leurs "dénonciations" successives, voir les actes d'un colloque: Le Malentendu: généalogie du geste herméneutique, textes réunis par Bruno Clément et Marc Escola, Saint-Denis, Presses Universitaires de Vincennes, 2003. Je tiens néanmoins à marquer ici mon désaccord avec les postulats posés les éditeurs dans leur "présentation", p. 5-14. Ceux-ci pensent pouvoir fonder une position herméneutique radicalement "relativiste" sur le prétendu constat que toute interprétation nouvelle (et/ou réfutation d'une erreur passée) ne serait, dans les sciences humaines, que le symptôme de la nécessité pour l'interprète de s'imposer, au sein de l'institution universitaire, aux dépens de ses prédécesseurs et/ou collègues. Bâtie sur des sociologismes psychologisants que rien ne vient étayer, cette position semble viser à deux choses : rendre toute interprétation, aussi farfelue soit-elle, légitime comme par défaut et interdire corrélativement (mais de quel droit?) aux membres d'une communauté disciplinaire de se réclamer d'une conception « cumulative » de leurs pratiques, où - sans qu'on ait nécessairement besoin d'aligner le fonctionnement sciences humaines sur celui des discours dits «scientifiques »- l'exactitude resterait néanmoins une valeur.

71. Rettorica e Poetica, op. cit., p. 165.

72. La diffusion du commentaire de Robortello en France fut sans doute non négligeable : la bibliothèque de Richelieu contenait un exemplaire de ce texte (dans le catalogue de la BnF, l'exemplaire côté Y 4-5, publié à Bâle en 1555, porte les armes du Cardinal de Richelieu sur les plats et la tranche, ainsi que quelques marginalia d'une main $d u \mathrm{XVII}^{\mathrm{e}}$ ) et celle de chapelain également (voir Colbert Searles, Catalogue de tous les livres de feu M. Jean Chapelain, 1676 ?, Stanford University, 1912, p. 80, $\mathrm{n}^{\circ} 2782$ et 2788). Corneille semble l'avoir lu soigneusement.

73. Corneille, Discours de la tragédie, et des moyens de la traiter, selon le vraisemblable et le nécessaire, Euvres complètes, éditées par Georges Couton, Paris, Gallimard, "Bibliothèque de la Pléiade ", 1980-1987, 3 vol., tome III, p. 145-146.

74. Corneille fait allusion aux commentaires que Robortello donne des lignes 1453a 7-22 puis 1453b 1-8 de la Poétique, soient des passages qui mettent en avant comme vraiment tragique l'action qui fait advenir à un homme bon un malheur que seule l'erreur peut expliquer, produisant ainsi terreur et pitié chez les spectateurs (Explicationes, op. cit., p. 129-141). Robortello explique effectivement dans son commentaire qu'ÆEdipe roi est, selon lui, une des seules tragédies grecques susceptible de s'inscrire dans ce schéma. Cette norme n'a donc pu être mise en avant par Aristote que comme un modèle de la meilleure tragédie possible et non comme un règle devant s'appliquer à toutes (p. 133).

75. Pour une analyse de la place de la «force de l'exemple» dans le théâtre de Corneille, voir Déborah Blocker et Dinah Ribard, «Figures de l'instruction dans l'Illusion comique de Pierre Corneille » dans Lectures du jeune Corneille: L'Illusion comique et Le Cid, textes réunis par JeanYves Vialleton, Rennes, Presses Universitaires de Rennes, 2001, p. 127-142.

76. Je me permets de renvoyer à ma thèse, Usages de la Comédie: utilités et plaisirs de la représentation théâtrale dans la France du premier XVII siècle, Université de Paris-Sorbonne Nouvelle, 2001, A. N. R. T., Lille, 2003, ch. IV.

77. Pour l'analyse d'un exemple des plus contemporains, je me permets ici encore de renvoyer le lecteur à un de mes propres articles : «Aristote, star de la fiction hollywoodienne? », autour du livre de Michæl Tierno, Aristotle's Poetics for Screenwriters, Hyperion, New York, 2002, dans Critique, septembre 2003, $\mathrm{n}^{\circ}$ spécial American fiction, p. 650-657. 
78. Il suffit, pour s'en convaincre, de feuilleter la bibliographie compilée par O. J. Schrier, The Poetics of Aristotle and the Tractatus Coislinianus : a Bibliography from about 900 till 1996, Leiden, Brill, 1998.

79. Voir La Poétique, texte grec avec une traduction et des notes par Roselyne Dupont-Roc et Jean Lallot, préface de Tvzetan Todorov, Paris, Le Seuil, «coll. Poétique», 1980, p. 188-123. Pour d'autres énonciations françaises de cette lecture esthétisante de la «catharsis ", voir aussi P. Somville, Essai sur la Poétique d'Aristote et sur quelques aspects de sa postérité, Paris, Vrin, 1975, ch. 2 et La Poétique, introd., trad. nouvelle et notes de Michel Magnien, Paris, Le Livre de Poche, 1990, p. 40-43.

80. Sur cette notion, voir Jacques Rancière, Le Partage sensible : esthétique et politique, Paris, La Fabrique, 2000, p. 26-33.

81. Corneille, Trois discours sur le poème dramatique, édition présentée et annotée par Marc Escola et Bénédicte Louvat, Paris, « G-F », Flammarion, 1999, p. 45.

\section{AUTEUR \\ DÉBORAH BLOCKER}

Université de Pittsburgh (GRIHL) 